Draft VERSion August 31, 2018

Preprint typeset using LTEX style emulateapj v. 11/10/09

\title{
INCORPORATING KINETIC PHYSICS INTO A TWO-FLUID SOLAR-WIND MODEL WITH TEMPERATURE ANISOTROPY AND LOW-FREQUENCY ALFVÉN-WAVE TURBULENCE
}

\author{
Benjamin D. G. Chandran ${ }^{1}$, Timothy J. Dennis ${ }^{1}$, Eliot Quataert ${ }^{2}, \&$ Stuart D. Bale $^{3}$ \\ Draft version August 31, 2018
}

\begin{abstract}
We develop a 1D solar-wind model that includes separate energy equations for the electrons and protons, proton temperature anisotropy, collisional and collisionless heat flux, and an analytical treatment of low-frequency, reflection-driven, Alfvén-wave (AW) turbulence. To partition the turbulent heating between electron heating, parallel proton heating, and perpendicular proton heating, we employ results from the theories of linear wave damping and nonlinear stochastic heating. We account for mirror and oblique firehose instabilities by increasing the proton pitch-angle scattering rate when the proton temperature anisotropy exceeds the threshold for either instability. We numerically integrate the equations of the model forward in time until a steady state is reached, focusing on two fast-solar-wind-like solutions. These solutions are consistent with a number of observations, supporting the idea that AW turbulence plays an important role in the origin of the solar wind.
\end{abstract}

Subject headings: solar wind — Sun: corona — turbulence — waves

\section{INTRODUCTION}

The first theory for the origin of the solar wind was developed by E. N. Parker in the 1950s and 1960s. Parker (1958, 1965) based his analysis on a single-fluid, hydrodynamic model with electron thermal conduction. Although he obtained smooth, transonic solutions in agreement with some solar-wind observations, his work was unable to account for the large flow velocities and proton temperatures measured in fast-solar-wind streams near Earth. Subsequent studies generalized the Parker model to include separate energy equations for the protons and electrons (Hartle \& Sturrock 1968), temperature anisotropy (Leer \& Axford 1972; Whang 1972), super-radial expansion of the magnetic field (Holzer \& Leer 1980), collisionless heat flux (Hollweg 1974, 1976), and energy and momentum deposition by Alfvén waves (AWs) and AW turbulence (Alazraki \& Couturier 1971; Belcher \& Davis 1971; Hollweg 1973a b; Tu 1987, 1988).

The idea that AW turbulence plays an important role in the origin of the solar wind was originally proposed by Coleman (1968) and is consistent with a number of observations. For example, data obtained from the Solar Optical Telescope on the Hinode spacecraft reveal the presence of ubiquitous, AWlike motions in the low corona carrying an energy flux sufficient to power the solar wind (De Pontieu et al. 2007). Pervasive AW-like fluctuations are also seen at higher altitudes in the corona in observations from the Coronal Multichannel Polarimeter of the National Solar Observatory (Tomczyk et al. 2007). Voyager, Helios, Wind, and other spacecraft have measured broad-spectrum fluctuations in the magnetic field, electric field, and flow velocity in the solar wind, demonstrating that AW turbulence is present throughout the interplanetary medium (Tu \& Marsch 1995; Goldstein et al. 1995;

\footnotetext{
${ }^{1}$ Space Science Center and Department of Physics, University of New Hampshire, Durham, NH 03824; benjamin.chandran@unh.edu, tim.dennis@unh.edu

${ }^{2}$ Astronomy Department \& Theoretical Astrophysics Center, 601 Campbell Hall, The University of California, Berkeley, CA 94720; eliot@astro.berkeley.edu

${ }^{3}$ Physics Department \& Space Sciences Laboratory, 311 Old LeConte Hall, The University of California, Berkeley, CA 94720; bale@ssl.berkeley.edu
}

Bale et al. 2005). Moreover, the amplitudes of these turbulent fluctuations are sufficient to explain the heating rates that have been inferred from the proton and electron temperature profiles (Smith et al. 2001; MacBride et al. 2008; Cranmer et al. 2009; Stawarz et al. 2009). Radio-scintillation observations of solar-wind density fluctuations place upper limits on the turbulent heating rate in the solar wind that are consistent with solar-wind heating by AW turbulence (Harmon \& Coles 2005; Chandran et al 2009; but see also Spangler 2002). In addition, Faraday rotation of radio transmissions from the Helios spacecraft show that magnetic-field fluctuations in the corona at heliocentric distances between $2 R_{\odot}$ and $15 R_{\odot}$ are consistent with models in which the solar wind is driven by AW turbulence (Hollweg et al. 1982, 2010).

However, it is not clear that heating by AW turbulence can explain observations of ion temperature anisotropies. Measurements from the Helios spacecraft have shown that $T_{\perp p}>$ $T_{\| \mathrm{p}}$ in the core of the proton velocity distribution in low- $\beta_{\| \mathrm{p}}$ fast-wind streams, where

$$
\beta_{\| \mathrm{p}}=\frac{8 \pi n k_{\mathrm{B}} T_{\| \mathrm{p}}}{B^{2}}
$$

$n$ is the proton density, $k_{\mathrm{B}} T_{\| \mathrm{p}} / 2$ is the average energy per proton in thermal motions parallel to the magnetic field $\boldsymbol{B}$, and $k_{\mathrm{B}} T_{\perp \mathrm{p}}$ is the average energy per proton in thermal motions perpendicular to $\boldsymbol{B}$ (Marsch et al. 2004). Similarly, remote observations from the Ultraviolet Coronagraph Spectrometer (UVCS) on the Solar and Heliospheric Observatory have shown that $T_{\perp} \gg T_{\|}$for $\mathrm{O}^{+5}$ ions in coronal holes Kohl et al. 1998; Li et al. 1998; Antonucci et al. 2000). These observations pose a challenge to solar-wind models based on AW turbulence because the AW energy cascade is anisotropic, transporting AW energy primarily to small scales measured perpendicular to $\boldsymbol{B}$ and only weakly to small scales measured parallel to $B$ (Shebalin et al. 1983). As a consequence, very little energy cascades to high frequencies comparable to the ion cyclotron frequencies at which waves can dissipate via resonant cyclotron interactions (Ouataert 1998), which are the only route to perpendicular ion heating in a collisionless plasma within the framework of quasilinear theory (Stix 1992). 
A number of studies have gone beyond quasilinear theory to show that low-frequency AW turbulence can lead to perpendicular ion heating even in the absence of a cyclotron resonance (McChesney et al. 1987; Chen et al. 2001; Johnson \& Cheng 2001; Dmitruk et al. 2004; Voitenko \& Goossens 2004; Bourouaine et al. 2008; Parashar et al. 2009; Chandran et al. 2010; Chandran 2010). In this paper, we incorporate results from one of these studies (Chandran et al. 2010) into a quantitative solar-wind model to investigate the extent to which low-frequency AW turbulence can explain the observations of anisotropic proton temperatures discussed above. The model we have developed also includes the non-WKB reflection of AWs, proton and electron heat flux in both the collisional and collisionless regimes, and enhanced pitch-angle scattering when the proton temperature anisotropy is sufficiently large to excite mirror, cyclotron, or firehose instabilities. We describe this model in detail in Section 2 and discuss the numerical method we use to solve the equations of the model in Section 3 In Section 4 we present and analyze a steady-state, fast-wind-like solution and compare this solution to a number of observations. In Section 5 we present a second steady-state solution and discuss our results. We conclude in Section 6 and summarize the derivation of the equations of our model in the appendix.

\section{MATHEMATICAL FRAMEWORK}

In the solar wind, the Debye length $\lambda_{\mathrm{D}}$ and proton gyroradius $\rho_{\mathrm{p}}$ are vastly smaller than the length scales over which the bulk solar-wind properties vary appreciably. Because of this, the large-scale structure of solar-wind plasma can be rigorously described by taking the limit of the Vlasov and Maxwell equations in which $\rho_{p} \rightarrow 0$ and $\lambda_{D} \rightarrow 0$. This limit, described in more detail in the appendix, is some times referred to as Kulsrud's collisionless magnetohydrodynamics (Kulsrud 1983). This name is somewhat misleading, in that the resulting description is still kinetic in nature. In particular, two of the variables in Kulsrud's theory are the reduced proton and electron distribution functions, $f_{\mathrm{p}}$ and $f_{\mathrm{e}}$, which are independent of the gyrophase angle in velocity space and evolve in time according to the guiding-center Vlasov equation (Equation (A9).

We take Kulsrud's collisionless magnetohydrodynamics (MHD) as the starting point for our study, including the simplifying assumption that the plasma consists of just protons and electrons. We then follow Snyder et al. (1997) in adding a collision operator to the guiding-center Vlasov equation and taking velocity moments of this equation to obtain a hierarchy of fluid equations, as described in more detail in the appendix. To close this set of fluid equations for the protons, we set $f_{\mathrm{p}}$ equal to a bi-Maxwellian when evaluating the various fourth moments of $f_{\mathrm{p}}$. This approach offers a simple and, we expect, reasonably accurate way to solve for the proton heat flux in the presence of temperature anisotropy in both collisional and collisionless conditions. The same general equations (with differing treatments of the collision terms) were derived in different ways by Endeve \& Leen (2001), Lie-Svendsen et al. (2001), and Ramos (2003). For the electrons, we adopt the simplifying assumption that $f_{\mathrm{e}}$ is isotropic in velocity space and close the fluid equations by specifying the electron heat flux in terms of lower-order moments of $f_{\mathrm{e}}$. The most general versions of the resulting equations are given in the appendix. In this section, we specialize these equations to our 1D solarwind model and add terms to incorporate heating by AW wave turbulence, acceleration by the AW pressure force, and temperature isotropization by firehose and mirror instabilities.

To simplify the analysis, we neglect the Sun's rotation, take the background magnetic field to be fixed, and solve the fluid equations within a narrow, open magnetic flux tube centered on a radial magnetic field line. We follow Kopp \& Holzer (1976) in taking the cross sectional area of this flux tube to be

$$
a=a_{\odot}\left(\frac{r}{R_{\odot}}\right)^{2} f,
$$

where $r$ is heliocentric distance,

$$
f=\frac{f_{\max } e^{\left(r-R_{1}\right) / \sigma}+f_{1}}{e^{\left(r-R_{1}\right) / \sigma}+1},
$$

$f_{1}=1-\left(f_{\max }-1\right) \exp \left[\left(R_{\odot}-R_{1}\right) / \sigma\right]$, and $a_{\odot}, f_{\max }, R_{1}$, and $\sigma$ are constants. The function $f$ increases from 1 at $r=R_{\odot}$ to $f_{\max }$ at $r \gg R_{1}$, with most of the variation in $f$ occurring between $r=R_{1}-\sigma$ and $r=R_{1}+\sigma$. To reduce the number of free parameters, we set

$$
\sigma=R_{1}
$$

The inner radius of our model corresponds to the coronal base just above the transition region. We set this radius equal to $R_{\odot}$, neglecting the thickness of the chromosphere and transition region. By flux conservation, the strength of the magnetic field $\boldsymbol{B}$ satisfies

$$
B=\frac{B \odot a \odot}{a},
$$

where $B_{\odot}$ is the magnetic field strength at the coronal base. We take the cross sectional area at the coronal base, $a_{\odot}$, to be $\ll R_{\odot}^{2}$ and $\ll R_{1}^{2}$, so that the flux tube is thin. As a consequence, the magnetic field direction is approximately radial everywhere within the flux tube. We thus set

$$
\hat{b} \cdot \nabla \rightarrow \frac{\partial}{\partial r}
$$

where we have taken the magnetic field to be pointing away from the Sun. The condition $\boldsymbol{\nabla} \cdot \boldsymbol{B}=0$ then gives

$$
\boldsymbol{\nabla} \cdot \hat{\boldsymbol{b}}=\frac{1}{a} \frac{\partial a}{\partial r}
$$

We take the solar-wind outflow velocity to be everywhere parallel to the magnetic field,

$$
\boldsymbol{U}=U \hat{\boldsymbol{b}}
$$

and define the Lagrangian time derivative

$$
\frac{d}{d t}=\frac{\partial}{\partial t}+U \frac{\partial}{\partial r}
$$

There are eight dependent variables in our model: the proton (or electron) number density $n$, the proton (or electron) outflow velocity $U$, the electron temperature $T_{\mathrm{e}}$, the perpendicular and parallel proton temperatures $T_{\perp \mathrm{p}}$ and $T_{\| \mathrm{p}}$, the proton heat fluxes $q_{\perp \mathrm{p}}$ and $q_{\| \mathrm{p}}$, and the energy density of AWs propagating away from the Sun $\mathcal{E}_{\mathrm{w}}$. The proton heat flux $q_{\perp \mathrm{p}}$ is a flow along $\boldsymbol{B}$ of perpendicular proton kinetic energy; no heat flows across the magnetic field in the model. The eight dependent variables of the model depend upon time $t$ and a single spatial coordinate $r$ and satisfy the following eight equations:

$$
\frac{d n}{d t}=-\frac{n}{a} \frac{\partial}{\partial r}(a U)
$$




$$
\begin{gathered}
\frac{d U}{d t}=-\frac{k_{\mathrm{B}}}{\rho} \frac{\partial}{\partial r}\left[n\left(T_{\mathrm{e}}+T_{\| \mathrm{p}}\right)\right]+\frac{k_{\mathrm{B}}\left(T_{\perp \mathrm{p}}-T_{\| \mathrm{p}}\right)}{m_{\mathrm{p}} a} \frac{\partial a}{\partial r} \\
-\frac{G M_{\odot}}{r^{2}}-\frac{1}{2 \rho} \frac{\partial \mathcal{E}_{\mathrm{w}}}{\partial r}, \\
\frac{3}{2} n^{5 / 3} k_{\mathrm{B}} \frac{d}{d t}\left(\frac{T_{\mathrm{e}}}{n^{2 / 3}}\right)=Q_{\mathrm{e}}-\frac{1}{a} \frac{\partial}{\partial r}\left(a q_{\mathrm{e}}\right)+3 v_{\mathrm{pe}} n k_{\mathrm{B}}\left(T_{\mathrm{p}}-T_{\mathrm{e}}\right), \\
B n k_{\mathrm{B}} \frac{d}{d t}\left(\frac{T_{\perp \mathrm{p}}}{B}\right)=Q_{\perp \mathrm{p}}-\frac{1}{a^{2}} \frac{\partial}{\partial r}\left(a^{2} q_{\perp \mathrm{p}}\right)+\frac{1}{3} v_{\mathrm{p}} n k_{\mathrm{B}}\left(T_{\| \mathrm{p}}-T_{\perp \mathrm{p}}\right) \\
+2 v_{\mathrm{pe}} n k_{\mathrm{B}}\left(T_{\mathrm{e}}-T_{\perp \mathrm{p}}\right), \\
\frac{n^{3} k_{\mathrm{B}}}{2 B^{2}} \frac{d}{d t}\left(\frac{B^{2} T_{\| \mathrm{p}}}{n^{2}}\right)=Q_{\| \mathrm{p}}-\frac{1}{a} \frac{\partial}{\partial r}\left(a q_{\| \mathrm{p}}\right)+\frac{q_{\perp \mathrm{p}}}{a} \frac{\partial a}{\partial r} \\
+\frac{1}{3} v_{\mathrm{p}} n k_{\mathrm{B}}\left(T_{\perp \mathrm{p}}-T_{\| \mathrm{p}}\right)+v_{\mathrm{pe}} n k_{\mathrm{B}}\left(T_{\mathrm{e}}-T_{\| \mathrm{p}}\right), \\
n^{2} \frac{d}{d t}\left(\frac{q_{\perp \mathrm{p}}}{n^{2}}\right)=-\frac{n k_{\mathrm{B}}^{2} T_{\| \mathrm{p}}}{m_{\mathrm{p}}} \frac{\partial T_{\perp \mathrm{p}}}{\partial r}+\frac{n k_{\mathrm{B}}^{2} T_{\perp \mathrm{p}}\left(T_{\perp \mathrm{p}}-T_{\| \mathrm{p}}\right)}{m_{\mathrm{p}} a} \frac{\partial a}{\partial r} \\
-v_{\mathrm{p}} q_{\perp \mathrm{p}}, \\
\frac{n^{4}}{B^{3}} \frac{d}{d t}\left(\frac{B^{3} q_{\| \mathrm{p}}}{n^{4}}\right)=-\frac{3 n k_{\mathrm{B}}^{2} T_{\| \mathrm{p}}}{2 m_{\mathrm{p}}} \frac{\partial T_{\| \mathrm{p}}}{\partial r}-v_{\mathrm{p}} q_{\| \mathrm{p}},
\end{gathered}
$$

and (Dewar 1970)

$$
\frac{\partial \mathcal{E}_{\mathrm{W}}}{\partial t}+\frac{1}{a} \frac{\partial}{\partial r}\left[a\left(U+v_{\mathrm{A}}\right) \mathcal{E}_{\mathrm{w}}\right]+\frac{\mathcal{E}_{\mathrm{W}}}{2 a} \frac{\partial}{\partial r}(a U)=-Q,
$$

where

$$
v_{\mathrm{A}}=\frac{B}{\sqrt{4 \pi \rho}}
$$

is the Alfvén speed, $\rho$ is the mass density, and $M_{\odot}$ is the mass of the Sun. Since we are treating the solar wind as a protonelectron plasma, we ignore the contribution of alpha particles and other particle species to $\rho$ and set

$$
\rho=m_{\mathrm{p}} n \text {. }
$$

The quantities $Q_{\mathrm{e}}, Q_{\perp \mathrm{p}}$, and $Q_{\| \mathrm{p}}$ are, respectively, the electron heating rate, the perpendicular proton heating rate, and the parallel proton heating rate per unit volume from the dissipation of AW turbulence (Section 2.4),

$$
Q=Q_{\mathrm{e}}+Q_{\perp \mathrm{p}}+Q_{\| \mathrm{p}}
$$

is the total turbulent heating rate per unit volume, and $q_{\mathrm{e}}$ is the electron heat flux (Section 2.1). The quantity

$$
v_{\mathrm{pe}}=\frac{4 \sqrt{2 \pi m_{\mathrm{e}}} e^{4} n \ln \Lambda}{3 m_{\mathrm{p}}\left(k_{\mathrm{B}} T_{\mathrm{e}}\right)^{3 / 2}}
$$

is the Coulomb collision frequency for energy exchange between protons and electrons (Schunk 1975), where $m_{\mathrm{p}}$ and $m_{\mathrm{e}}$ are the proton and electron masses and $\ln \Lambda$ is the Coulomb logarithm, which we take to be 23 . We set

$$
v_{\mathrm{p}}=v_{\mathrm{pp}, \mathrm{C}}+\mathrm{v}_{\mathrm{inst}},
$$

where

$$
v_{\mathrm{pp}, \mathrm{C}}=\frac{4 \sqrt{\pi} e^{4} n \ln \Lambda}{3 \sqrt{m_{\mathrm{p}}}\left(k_{\mathrm{B}} T_{\mathrm{p}}\right)^{3 / 2}}
$$

is the proton-proton Coulomb collision frequency (Schunk 1975), $v_{\text {inst }}$ is a scattering rate associated with small-scale plasma waves that are excited when the proton temperature anisotropy becomes sufficiently large (Section 2.2), and

$$
T_{\mathrm{p}}=\frac{2 T_{\perp \mathrm{p}}+T_{\| \mathrm{p}}}{3} .
$$

Equation (10) expresses the conservation of mass in our 1D model. Equation (11) is the same as the momentum equation in Kulsrud's collisionless MHD (Equation (A2)), except that we have added the gravitational acceleration and the AW pressure force (Dewar 1970). In the absence of turbulent heating, heat flow, and collisions, the right-hand sides of Equations (12) through (14) vanish. In this case, the Lagrangian time derivative of the electron specific entropy $\left(\propto \ln \left(T_{\mathrm{e}} / n^{2 / 3}\right)\right)$ vanishes, and the protons obey the doubleadiabatic theory of Chew, Goldberger, \& Low (1956). When $v_{\mathrm{p}}$ becomes sufficiently large, $T_{\perp \mathrm{p}} \simeq T_{\| \mathrm{p}}$ and the collisional terms in Equations (15) and (16) (which are $\propto v_{\mathrm{p}}$ ) are much larger than the left-hand sides of these equations. In this limit, $q_{\perp \mathrm{p}}$ and $q_{\| \mathrm{p}}$ are determined from Equations (15) and (16) by balancing the collisional terms against the source terms (which contain $T_{\perp \mathrm{p}}$ and/or $T_{\| \mathrm{p}}$ but not $q_{\perp \mathrm{p}}$ or $q_{\| \mathrm{p}}$ ), and the total proton heat flux $q_{\perp \mathrm{p}}+q_{\| \mathrm{p}}$ becomes approximately equal to the proton heat flux in collisional transport theory (Braginskii 1965).

Upon multiplying Equation (11) by $\rho U$ and adding the resulting equation to the sum of Equations (12), (13), (14), and (17), we obtain a total energy equation,

$$
\frac{\partial \mathcal{E}_{\mathrm{tot}}}{\partial t}+\frac{1}{a} \frac{\partial}{\partial r}\left(a F_{\mathrm{tot}}\right)=0
$$

where

$$
\mathcal{E}_{\text {tot }}=\frac{\rho U^{2}}{2}-\frac{G M_{\odot} \rho}{r}+n k_{\mathrm{B}}\left(\frac{3 T_{\mathrm{e}}}{2}+T_{\perp \mathrm{p}}+\frac{T_{\| \mathrm{p}}}{2}\right)+\mathcal{E}_{\mathrm{w}}
$$

is the total energy density, and

$$
\begin{gathered}
F_{\text {tot }}=\frac{\rho U^{3}}{2}-\frac{U G M_{\odot} \rho}{r}+U n k_{\mathrm{B}}\left(\frac{5 T_{\mathrm{e}}}{2}+T_{\perp \mathrm{p}}+\frac{3 T_{\| \mathrm{p}}}{2}\right) \\
+q_{\mathrm{e}}+q_{\perp \mathrm{p}}+q_{\| \mathrm{p}}+\left(\frac{3 U}{2}+v_{\mathrm{A}}\right) \mathcal{E}_{\mathrm{w}}
\end{gathered}
$$

is the total energy flux. In steady state, $a F_{\text {tot }}$ is independent of $r$, and the total flow of energy into the base of the flux tube equals the total flow of energy through the flux-tube cross section at all other radii.

\subsection{Electron Heat Flux}

Close to the Sun, $n$ is sufficiently large that the electron Coulomb mean free path,

$$
\lambda_{\mathrm{mfp}}=\frac{\sqrt{k_{\mathrm{B}} T_{\mathrm{e}} / m_{\mathrm{e}}}}{v_{\mathrm{e}}},
$$


is much shorter than the radial distance $l_{T}=T_{\mathrm{e}} /\left(\partial T_{\mathrm{e}} / \partial r\right)$ over which $T_{\mathrm{e}}$ varies appreciably. The quantity

$$
v_{\mathrm{e}}=2.9 \times 10^{-6}\left(\frac{n}{1 \mathrm{~cm}^{-3}}\right)\left(\frac{k_{\mathrm{B}} T_{\mathrm{e}}}{1 \mathrm{eV}}\right)^{-3 / 2} \ln \Lambda \mathrm{s}^{-1}
$$

is the electron collision frequency (Book 1983). We expect the electron heat flux in this near-Sun region to be approximately equal to the Spitzer value (Spitzer \& Härm 1953),

$$
\boldsymbol{q}_{\mathrm{e}, \mathrm{S}}=-\kappa_{\mathrm{e} 0} T_{\mathrm{e}}^{5 / 2}\left(\hat{\boldsymbol{b}} \cdot \nabla T_{\mathrm{e}}\right) \hat{\boldsymbol{b}}
$$

where

$$
\kappa_{\mathrm{e} 0}=\frac{1.84 \times 10^{-5}}{\ln \Lambda} \operatorname{erg~s}^{-1} \mathrm{~K}^{-7 / 2} \mathrm{~cm}^{-1}
$$

Farther from the Sun, $\lambda_{\mathrm{mfv}} \gtrsim l_{T}$, and $q_{\mathrm{e}}$ deviates from the Spitzer value. We follow Hollweg (1974, 1976) in taking the collisionless heat flux in this region to be approximately

$$
\boldsymbol{q}_{\mathrm{e}, \mathrm{H}}=\frac{3}{2} \alpha_{\mathrm{H}} U n k_{\mathrm{B}} T_{\mathrm{e}} \hat{\boldsymbol{b}}
$$

where $\alpha_{\mathrm{H}}$ is a constant that we treat as a free parameter. Hollweg (1976) argued that the transition between the collisional and collisionless regimes occurs at the radius at which $\lambda_{\text {mfp }} \simeq 0.5 r$. To interpolate smoothly between the two regimes, we set the electron heat flux equal to

$$
\boldsymbol{q}_{\mathrm{e}}=\psi \boldsymbol{q}_{\mathrm{e}, \mathrm{H}}+(1-\psi) \boldsymbol{q}_{\mathrm{e}, \mathrm{S}}
$$

where

$$
\psi=\frac{\left(r / r_{\mathrm{H}}\right)^{2}}{1+\left(r / r_{\mathrm{H}}\right)^{2}}
$$

and $r_{\mathrm{H}}$ is a constant that we choose to coincide with the radius at which $\lambda_{\mathrm{mfp}}=0.5 \mathrm{r}$. For the numerical solutions presented in Sections 4 and 5.7, we set $r_{\mathrm{H}}=5 R_{\odot}$, and confirm post facto that $\lambda \simeq 0.5 r$ at $r=r_{\mathrm{H}}$ (see Figure6).

\subsection{Proton Pitch-Angle Scattering from Firehose and Mirror Instabilities}

If the proton temperature-anisotropy ratio

$$
R=\frac{T_{\perp \mathrm{p}}}{T_{\| \mathrm{p}}}
$$

becomes either too large or too small, the plasma becomes unstable. Spacecraft measurements show that the values of $R$ found in the solar wind are bounded from below by the instability threshold of the oblique firehose mode and from above by the instability threshold of the mirror mode (Kasper et al. 2002; Hellinger et al. 2006; Bale et al. 2009). In particular, most of the measured values of $R$ correspond to plasma parameters for which $\gamma_{\max }<10^{-3} \Omega_{\mathrm{p}}$, where $\gamma_{\max }$ is the maximum growth rate of the oblique firehose or mirror instability and $\Omega_{\mathrm{p}}$ is the proton cyclotron frequency. The value of $R$ for which $\gamma_{\max }=10^{-3} \Omega_{\mathrm{p}}$ is approximately

$$
R_{\mathrm{m}}=1+0.77\left(\beta_{\| \mathrm{p}}+0.016\right)^{-0.76}
$$

for the mirror instability, and approximately

$$
R_{\mathrm{f}}=1-1.4\left(\beta_{\| \mathrm{p}}+0.11\right)^{-1}
$$

for the oblique firehose instability (Hellinger et al. 2006).
Presumably, when the plasma becomes unstable, smallscale electromagnetic fluctuations grow and enhance the proton pitch-angle scattering rate, preventing the temperature anisotropy from increasing further. We incorporate this effect into our model through the term $v_{\text {inst }}$ in Equation (22), with

$$
v_{\text {inst }}=v_{0} \exp \left[\frac{12\left(R-R_{\mathrm{m}}\right)}{R_{\mathrm{m}}}\right]+\mathrm{v}_{0} \exp \left[\frac{12\left(\bar{R}_{\mathrm{f}}-R\right)}{\bar{R}_{\mathrm{f}}}\right],
$$

$v_{0}=0.02 \sqrt{G M_{\odot} / R_{\odot}^{3}}$, and $\bar{R}_{\mathrm{f}}=\max \left(R_{\mathrm{f}}, 10^{-6}\right)$. A similar approach was employed by Sharma et al. (2006) in numerical simulations of accretion flows around black holes.

\subsection{Alfvén Wave Turbulence}

The Sun launches different types of waves that propagate outward into the solar atmosphere. In our model, we retain only the non-compressive Alfvén wave (AW), in part for simplicity and in part because the AW is the most promising wave type for transporting energy over large distances into the corona and solar wind (Barnes 1966; Velli et al. 1989; Matthaeus et al. 1999; Suzuki \& Inutsuka 2005; Cranmer \& van Ballegooijen 2005). For AW fluctuations, the fluctuating velocity vector $\delta v$ and magnetic field vector $\delta \boldsymbol{B}$ lie in the plane perpendicular to $\boldsymbol{B}_{0}$. We define the Elsassër variables

$$
z^{ \pm}=\delta v \mp \frac{\delta B}{\sqrt{4 \pi \rho}}
$$

and, as mentioned previously, take $\boldsymbol{B}_{0}$ to point away from the Sun. In the small-amplitude limit, $\boldsymbol{z}^{+}$fluctuations are AWs that propagate with an outward radial velocity of $U+v_{\mathrm{A}}$, while the $\boldsymbol{z}^{-}$fluctuations are AWs that propagate with a radial velocity $U-v_{\mathrm{A}}$. Near the Sun, $U<v_{\mathrm{A}}$ and $z^{-}$fluctuations propagate towards smaller $r$.

To a good approximation, AWs in the solar corona and solar wind can be described within the framework of reduced MHD (Kadomtsev \& Pogutse 1974; Strauss 1976; Zank \& Matthaeus 1992; Schekochihin et al. 2009). In reduced MHD, the outward-propagating $z^{+}$waves generated by the Sun do not interact with one another. However, we assume that most of the AW energy is at periods of tens of minutes to hours, which makes the wavelengths in the radial direction sufficiently long that the AWs undergo significant non-WKB reflection, converting some of the $z^{+}$waves into $z^{-}$waves (Heinemann \& Olbert 1980; Velli 1993). Interactions between $z^{+}$and $z^{-}$fluctuations then cause wave energy to cascade from large scales to small scales (Iroshnikov 1963; Kraichnan 1965; Velli et al. 1989; Matthaeus et al. 1999; van Ballegooijen et al. 2011). At sufficiently small scales, the $z^{ \pm}$fluctuations dissipate. Although some reflection occurs, we assume that

$$
z^{-} \ll z^{+}
$$

and neglect the contribution of $z^{-}$to the wave energy density $\mathcal{E}_{\mathrm{w}}$, which is then given by

$$
\mathcal{E}_{\mathrm{w}}=\frac{\rho\left(z_{\mathrm{rms}}^{+}\right)^{2}}{4}
$$

where $z_{\mathrm{rms}}^{+}$is the rms amplitude of $z^{+}$fluctuations.

To describe the cascade of wave energy in the presence of wave reflections, we adopt the phenomenological 
model of Dmitruk et al. (2002), which was later extended by Chandran \& Hollweg (2009) to account for the solar-wind outflow velocity. The essence of these models is to balance the rate at which $z^{-}$waves are produced by wave reflections against the rate at which the $z^{-}$waves cascade and dissipate via interactions with $z^{+}$waves. This balance leads to the following estimate for the rms amplitude of $z^{-}$(Chandran \& Hollweg 2009):

$$
z_{\mathrm{rms}}^{-}=\frac{L_{\perp}\left(U+v_{\mathrm{A}}\right)}{v_{\mathrm{A}}}\left|\frac{\partial v_{\mathrm{A}}}{\partial r}\right|,
$$

where $L_{\perp}$ is the correlation length (outer scale) of the Alfvénic fluctuations in the plane perpendicular to $\boldsymbol{B}_{0}$. The rate at which energy cascades and dissipates per unit volume is then

$$
Q=\frac{c_{\mathrm{d}} \rho z_{\mathrm{rms}}^{-}\left(z_{\mathrm{rms}}^{+}\right)^{2}}{4 L_{\perp}}
$$

where $c_{\mathrm{d}}$ is a dimensionless number. Since our estimate of $z_{\mathrm{rms}}^{-}$is proportional to $L_{\perp}$, the value of $Q$ in equation (43) is independent of $L_{\perp}$. Because of Equation (40), we have omitted a term $\propto z_{\text {rms }}^{+}\left(z_{\text {rms }}^{-}\right)^{2}$ that is some times included in the turbulent heating rate (Hossain et al. 1995).

\subsection{Proton and Electron Heating Rates}

In Equation (17), the rate $Q$ at which energy is drained from the AWs equals the energy cascade rate given in Equation (43), which is determined by the "large-scale quantities" $z_{\mathrm{rms}}^{+}, z_{\mathrm{rms}}^{-}$, and $L_{\perp}$. All of the AW energy that cascades to small scales dissipates, contributing to turbulent heating, but the way that $Q$ is apportioned between $Q_{\mathrm{e}}, Q_{\perp \mathrm{p}}$, and $Q_{\| \mathrm{p}}$ depends upon the mechanisms that dissipate the fluctuations at length scales $\ll L_{\perp}$. In this section, we describe how we divide the turbulent heating power between $Q_{\mathrm{e}}, Q_{\perp \mathrm{p}}$, and $Q_{\| \mathrm{p}}$ using results from the theories of linear wave damping and nonlinear stochastic heating.

Nonlinear interactions between counter-propagating AWs cause AW energy to cascade primarily to larger $k_{\perp}$ and only weakly to larger $\left|k_{\|}\right|$, where $k_{\perp}$ and $k_{\|}$are wavevector components perpendicular and parallel to $\boldsymbol{B}_{0}$ (Shebalin et al. 1983; Goldreich \& Sridhar 1995; Ng \& Bhattacharjee 1996; Galtier et al. 2000). This cascade does not transfer AW energy efficiently to higher frequencies (the AW frequency being $k_{\|} v_{\mathrm{A}}$ ), and thus cyclotron damping is not an important dissipation mechanism for the anisotropic AW cascade Cranmer \& van Ballegooijen 2003; Howes et al. 2008a). There may be other mechanisms in the solar wind that generate AWs with sufficiently high frequencies that the AWs undergo cyclotron damping (Leamon et al. 1998; Hamilton et al. 2008), such as a turbulent cascade involving compressive waves (Chandran 2005, 2008b; Yoon \& Fang 2008) or instabilities driven by proton or alpha-particle beams (Gomberoff et al. 1996; Hellinger \& Trávníček 2011). However, we do not account for these possibilities in our model.

When AW energy cascades to $k_{\perp} \rho_{\mathrm{p}} \simeq 1$, the cascade transitions to a kinetic Alfvén wave (KAW) cascade (Bale et al. 2005; Howes et al. 2008a b; Schekochihin et al. 2009; Sahraoui et al. 2009), and the KAW fluctuations undergo Landau damping and transit-time damping (Ouataert 1998; Gruzinov 1998; Leamon et al. 1999) and dissipation via stochastic heating (McChesney et al. 1987; Chen et al. 2001; Johnson \& Cheng 2001). Some of the turbulent energy dissipates at $k_{\perp} \rho_{\mathrm{p}} \simeq 1$, and some of the turbulent energy cascades to, and then dissipates at, smaller scales. Before describing the details of how we incorporate dissipation into our model, we first summarize our general approach. We make the approximation that the dissipation occurs in two distinct wavenumber ranges: $k_{\perp} \rho_{\mathrm{p}} \sim 1$ and $k_{\perp} \rho_{\mathrm{p}} \gg 1$. We divide the total dissipation power between these two wavenumber ranges by comparing the energy cascade time scale and damping time scale at $k_{\perp} \rho_{\mathrm{p}}=1$ (see Equation (53) below). We divide the power that is dissipated at $k_{\perp} \rho_{\mathrm{p}} \sim 1$ between $Q_{\mathrm{e}}, Q_{\perp \mathrm{p}}$, and $Q_{\| \mathrm{p}}$ by comparing the damping rates at $k_{\perp} \rho_{\mathrm{p}}=1$ associated with three different dissipation mechanisms, each of which contributes primarily to either $Q_{\mathrm{e}}, Q_{\perp \mathrm{p}}$, or $Q_{\| \mathrm{p}}$. We then assume that all of the power that dissipates at $k_{\perp} \gg \rho_{\mathrm{p}}^{-1}$ does so via interactions with electrons, thereby contributing to $Q_{\mathrm{e}}$.

We define $\gamma_{\mathrm{e}}$ and $\gamma_{\mathrm{p}}$ to be the electron and proton contributions to the linear damping rate of KAWs at $k_{\perp} \rho_{\mathrm{p}}=1$, where $\rho_{\mathrm{p}}$ is the proton gyroradius. Using a numerical code that solves the full hot-plasma dispersion relation (Quataert 1998), we have calculated $\gamma_{\mathrm{e}}$ and $\gamma_{\mathrm{p}}$ for a range of plasma parameters, assuming isotropic proton and electron temperatures. For $10^{-3}<\beta_{\mathrm{p}}<10,1 \lesssim T_{\mathrm{p}} / T_{\mathrm{e}} \lesssim 5$, and $\left|k_{\|} v_{\mathrm{A}}\right| \ll \Omega_{\mathrm{p}}$, our results are well approximated by the following formulas:

$$
\frac{\gamma_{\mathrm{e}}}{\left|k_{\|} v_{\mathrm{A}}\right|}=0.01\left(\frac{T_{\mathrm{e}}}{T_{\mathrm{p}} \beta_{\mathrm{p}}}\right)^{1 / 2}\left[\frac{1+0.17 \beta_{\mathrm{p}}^{1.3}}{1+\left(2800 \beta_{\mathrm{e}}\right)^{-1.25}}\right]
$$

and

$$
\frac{\gamma_{\mathrm{p}}}{\left|k_{\|} v_{\mathrm{A}}\right|}=0.08\left(\frac{T_{\mathrm{e}}}{T_{\mathrm{p}}}\right)^{1 / 4} \beta_{\mathrm{p}}^{0.7} \exp \left(-\frac{1.3}{\beta_{\mathrm{p}}}\right),
$$

where $\beta_{\mathrm{p}}=8 \pi n k_{\mathrm{B}} T_{\mathrm{p}} / B_{0}^{2}$ and $\beta_{\mathrm{e}}=8 \pi n k_{\mathrm{B}} T_{\mathrm{e}} / B_{0}^{2}$. In Figure 1 we compare Equations (44) and (45) with our numerical solutions for the case in which $T_{\mathrm{p}}=2 T_{\mathrm{e}}$.

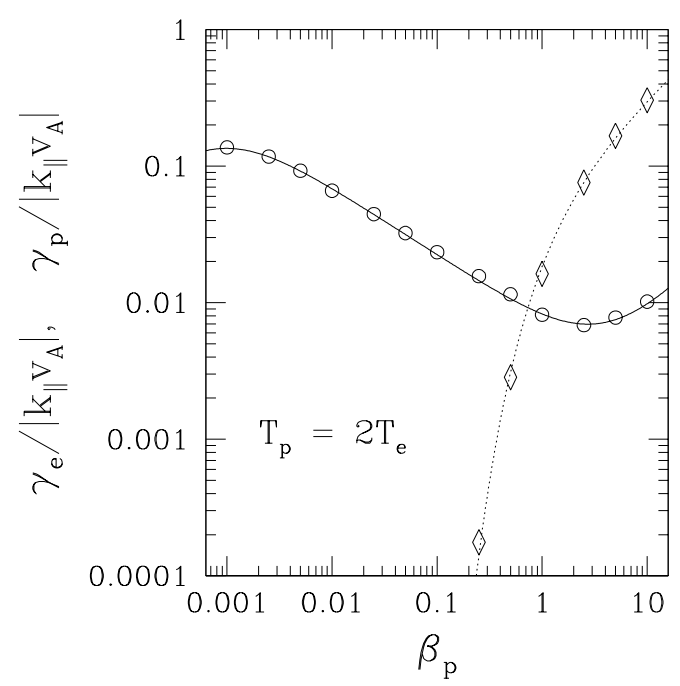

FIG. 1.- Solid line gives the electron contribution to the KAW damping rate at $k_{\perp} \rho_{\mathrm{p}}=1$ from Equation (44), and the dotted line gives the proton contribution to the KAW damping rate at $k_{\perp} \rho_{\mathrm{p}}=1$ from Equation 455. The circles and diamonds are, respectively, the electron and proton contributions to the KAW damping rate at $k_{\perp} \rho_{\mathrm{p}}=1$ in our numerical solutions to the full hot-plasma dispersion relation for Maxwellian electrons and protons. 
At $k_{\perp} \rho_{\mathrm{p}} \simeq 1, \mathrm{AW} / \mathrm{KAW}$ turbulence has a range of $k_{\|}$values. However, we approximate the linear proton and electron damping rates by assigning a single effective $\left|k_{\|}\right|$to the spectrum at $k_{\perp} \rho_{\mathrm{p}}=1$ given by the criticalbalance condition (Higdon 1984; Goldreich \& Sridhar 1995; Cho \& Lazarian 2004; Boldyrev 2006)

$$
\left|k_{\|}\right| v_{\mathrm{A}}=t_{\mathrm{c}}^{-1}
$$

where

$$
t_{\mathrm{c}}=\frac{\rho \delta v_{\mathrm{p}}^{2}}{Q}
$$

is the energy cascade time at $k_{\perp} \rho_{\mathrm{p}}=1$, and $\delta v_{\mathrm{p}}$ is the rms amplitude of AW/KAW velocity fluctuations at $k_{\perp} \rho_{\mathrm{p}} \sim 1$. In writing Equation (47), we have taken the total fluctuation energy per unit volume at $k_{\perp} \rho_{\mathrm{p}} \sim 1$ to be twice the kinetic energy density $\rho \delta v_{\mathrm{p}}^{2} / 2$, and we have assumed that dissipation at $k_{\perp}<\rho_{\mathrm{p}}^{-1}$ does not reduce the cascade power at $k_{\perp} \rho_{\mathrm{p}} \sim 1$ much below the level that is present throughout the inertial range. There is some evidence that the magnetic fluctuations in the solar wind are consistent with turbulence theories based on critical balance (Horbury et al. 2008; Podesta 2009; Forman et al. 2011). However, there are conflicting claims in the literature over the validity of Equation (46) when $z_{\mathrm{rms}}^{+} \gg z_{\mathrm{rms}}^{-}$, a point to which we return in Section 5.4

We assume that for length scales $\lambda$ between $\rho_{p}$ and the perpendicular AW correlation length (outer scale) $L_{\perp}$, the rms amplitude of the AW velocity fluctuations at perpendicular scale $\lambda$ is $\propto \lambda^{1 / 4}$ as in observations at $r=1$ AU (Podesta et al. 2007; Chen et al. 2011), direct numerical simulations of AW turbulence in the presence of a strong background magnetic field (Maron \& Goldreich 2001; Müller \& Grappin 2005; Mason et al. 2006; Perez \& Boldyrev 2008), and recent theories of strong AW turbulence (Boldyrev 2006; Perez \& Boldyrev 2009). We thus take

$$
\delta v_{\mathrm{p}}=\frac{z_{\mathrm{rms}}^{+}}{2}\left(\frac{\rho_{\mathrm{p}}}{L_{\perp}}\right)^{1 / 4} .
$$

We set

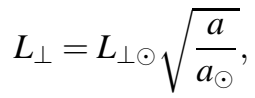

where $L_{\perp \odot}$ is the value of $L_{\perp}$ at the coronal base, so that $L_{\perp}$ increases in proportion to the cross-sectional radius of the flux tube in our model.

In addition to linear damping, KAWs at $k_{\perp} \rho_{\mathrm{p}} \sim 1$ undergo nonlinear damping through the "stochastic heating" of protons (McChesney et al. 1987; Chen et al. 2001; Johnson \& Cheng 2001). In stochastic proton heating, AW/KAW fluctuations at $k_{\perp} \rho_{\mathrm{p}} \sim 1$ cause proton orbits in the plane perpendicular to $\boldsymbol{B}_{0}$ to become stochastic, and the protons are subsequently energized by the time-varying electrostatic potential. Using numerical simulations of test particles interacting with a spectrum of randomly phased AWs and KAWs, Chandran et al. (2010) found that the effective damping rate of KAWs at $k_{\perp} \rho_{\mathrm{p}} \sim 1$ and $\beta_{\| \mathrm{p}} \lesssim 1$ from stochastic proton heating is

$$
\gamma_{\mathrm{s}}=0.18 \varepsilon_{\mathrm{p}} \Omega_{\mathrm{p}} \exp \left(-\frac{c_{2}}{\varepsilon_{\mathrm{p}}}\right)
$$

where $c_{2}$ is a dimensionless constant,

$$
\varepsilon_{\mathrm{p}}=\frac{\delta v_{\mathrm{p}}}{v_{\perp \mathrm{p}}}
$$

and $v_{\perp \mathrm{p}}=\sqrt{2 k_{\mathrm{B}} T_{\perp \mathrm{p}} / m_{\mathrm{p}}}$ is the proton perpendicular thermal speed. Chandran et al. (2010) found that $c_{2}=0.34$ for randomly phased AWs and KAWs, but conjectured that $c_{2}$ is smaller (and hence stochastic heating is more effective) in strong AW/KAW turbulence, because much of the dissipation in strong AW/KAW turbulence occurs within coherent structures in which the fluctuation amplitudes are larger than their rms values (Dmitruk et al. 2004). In the vicinity of such structures, proton orbits are more stochastic than on average, allowing for more efficient stochastic heating. Chandran (2010) developed a model of ion temperatures in coronal holes based on stochastic heating, and this model matched the observed $\mathrm{O}^{+5}$ temperature profile when $c_{2}$ was set equal to 0.15 . In our model, we leave $c_{2}$ as a free parameter.

The total effective damping rate of KAWs at $k_{\perp} \rho_{\mathrm{p}}=1$ is

$$
\gamma_{\mathrm{tot}}=\gamma_{\mathrm{e}}+\gamma_{\mathrm{p}}+\gamma_{\mathrm{s}}
$$

We define $\Gamma$ to be the fraction of the cascade power that is dissipated at $k_{\perp} \rho_{\mathrm{p}} \sim 1$. This fraction is roughly $\gamma_{\text {tot }} t_{\mathrm{c}}$ when $\gamma_{\text {tot }} t_{\mathrm{c}} \ll 1$ and roughly 1 when $\gamma_{\text {tot }} t_{\mathrm{c}} \gg 1$. To interpolate smoothly between these limits, we set

$$
\Gamma=\frac{\gamma_{\mathrm{tot}} t_{\mathrm{c}}}{1+\gamma_{\mathrm{tot}} t_{\mathrm{c}}}
$$

Landau damping and transit-time damping of KAWs on protons contribute to $Q_{\| \mathrm{p}}$ but not to $Q_{\perp \mathrm{p}}$ (Stix 1992). On the other hand, stochastic heating leads primarily to perpendicular proton heating when $\beta_{\| \mathrm{p}} \ll 1$ (Chandran et al. 2010). Johnson \& Cheng (2001) have shown that stochastic heating leads to significant perpendicular proton heating even at $\beta_{\| \mathrm{p}} \sim 1$, and for simplicity we take stochastic heating to contribute only to $Q_{\perp \mathrm{p}}$, regardless of the value of $\beta_{\| \mathrm{p}}$. We divide the cascade power that is dissipated at $k_{\perp} \rho_{\mathrm{p}} \sim 1$ into three parts - electron heating, perpendicular proton heating, and parallel proton heating — in proportion to the corresponding damping rates, $\gamma_{\mathrm{e}}, \gamma_{\mathrm{s}}$, and $\gamma_{\mathrm{p}}$. As mentioned previously, we assume that the fraction of the cascade power that is not dissipated at $k_{\perp} \rho_{\mathrm{p}} \sim 1$ cascades to scales $\ll \rho_{\mathrm{p}}$, dissipates via interactions with electrons, and contributes to $Q_{\mathrm{e}}$. This procedure leads to the relations

$$
\begin{gathered}
Q_{\mathrm{e}}=\frac{\left(1+\gamma_{\mathrm{e}} t_{\mathrm{c}}\right) Q}{1+\gamma_{\mathrm{tot}} t_{\mathrm{c}}}, \\
Q_{\perp \mathrm{p}}=\frac{\gamma_{\mathrm{s}} t_{\mathrm{c}} Q}{1+\gamma_{\mathrm{tot}} t_{\mathrm{c}}},
\end{gathered}
$$

and

$$
Q_{\| \mathrm{p}}=\frac{\gamma_{\mathrm{p}} t_{\mathrm{c}} Q}{1+\gamma_{\mathrm{tot}} t_{\mathrm{c}}}
$$

\section{NUMERICAL METHOD}

We integrate Equations (10) through (17) forward in time until a steady state is reached using the implicit numerical method described by Hu et al. (1997). We use a logarithmic grid in $r$ with grid points $r_{i}$ extending from $1 R_{\odot}$ to $1.2 \mathrm{AU}$, where $i=0,1,2, \ldots, N+1$ and $N=1320$. At each time step, we update the variables by integrating the equations only at $r_{1}, r_{2}, \ldots, r_{N}$. We then update the variables at $r_{0}$ and $r_{N+1}$ 

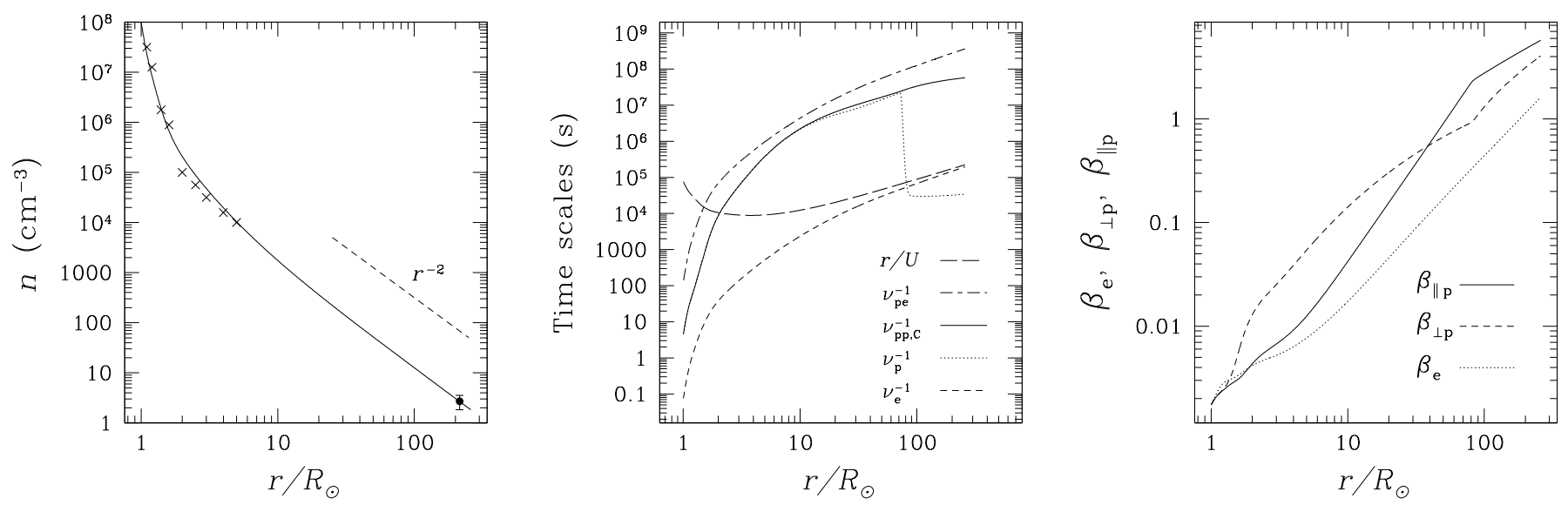

FIG. 2. - Left panel: Proton (or electron) number density in model (solid line). The $\times \mathrm{s}$ show observed values in a polar coronal hole near solar minimum from Table 14.19 of Allen (1973). The filled circle is the mean proton density $n=2.7 \pm 0.86 \mathrm{~cm}^{-3}$ at heliographic latitudes $>36^{\circ}$ measured during Ulysses' first polar orbit, scaled to $r=1 \mathrm{AU}$ (McComas et al. 2000). Middle panel: Important time scales in our steady-state numerical solution. Right panel: The ratios of thermal to magnetic pressure: $\beta_{\| \mathrm{p}}=8 \pi n k_{\mathrm{B}} T_{\| \mathrm{p}} / B^{2}, \beta_{\perp \mathrm{p}}=8 \pi n k_{\mathrm{B}} T_{\perp \mathrm{p}} / B^{2}$, and $\beta_{\mathrm{e}}=8 \pi n k_{\mathrm{B}} T_{\mathrm{e}} / B^{2}$.

using the following boundary conditions. At $r=r_{0}$ we set $n=n_{\odot}, T_{e}=T_{\perp \mathrm{p}}=T_{\| \mathrm{p}}=T_{\odot}$, and $\mathcal{E}_{\mathrm{w}}=n_{\odot} m_{\mathrm{p}}\left(\delta v_{\odot}\right)^{2}$, where $n_{\odot}, T_{\odot}$, and $\delta v_{\odot}$ are constants (see Table 1 ). We determine $U, q_{\perp \mathrm{p}}$, and $q_{\| \mathrm{p}}$ at $r=r_{0}$ by linearly extrapolating from the values at $r=r_{1}$ and $r=r_{2}$. We determine $U\left(r_{0}\right)$ in this way rather than by fixing the value of $U\left(r_{0}\right)$ so that the variables can evolve towards a steady-state transonic solution that passes smoothly through the sonic point. We determine $q_{\perp p}$ and $q_{\| \mathrm{p}}$ at $r=r_{0}$ by linear extrapolation for the following reason. At radii slightly greater than $r_{0}$, the values of $q_{\perp p}$ and $q_{\| \mathrm{p}}$ are determined to a good approximation by neglecting the terms on the left-hand sides of Equations (15) and (16), because the collision time $v_{\mathrm{p}}^{-1}$ is much shorter than the expansion time $r / U$. We call the values of $q_{\perp \mathrm{p}}$ and $q_{\| \mathrm{p}}$ determined in this way the "collisional values." If boundary conditions were imposed on $q_{\perp \mathrm{p}}$ and $q_{\| \mathrm{p}}$ at $r=r_{0}$ that were different from the collisional values, then in steady state a boundary layer would develop at $r=r_{0}$ of thickness $\sim U_{0} / v_{\mathrm{p} 0}$, where $U_{0}=U\left(r_{0}\right)$ and $v_{\mathrm{p} 0}=v_{\mathrm{p}}\left(r_{0}\right)$, and $q_{\perp \mathrm{p}}$ and $q_{\| \mathrm{p}}$ would approach their collisional values at $r \sim r_{0}+U_{0} / \mathrm{v}_{\mathrm{p} 0}$. However, by linearly extrapolating the values of $q_{\perp \mathrm{p}}$ and $q_{\| \mathrm{p}}$ to $r=r_{0}$, we prevent such unphysical boundary layers from appearing. At $r=r_{N+1}$ we evaluate all variables by linearly extrapolating from their values at $r=r_{N-1}$ and $r=r_{N}$. For the solutions presented in Sections 4 and 5.7 we used the following initial conditions: $T_{\mathrm{e}}=T_{\perp \mathrm{p}}=T_{\| \mathrm{p}}=T_{\odot}\left(3-2 R_{\odot} / r\right)\left(r / R_{\odot}\right)^{-2 / 7}$, $U=(655 \mathrm{~km} / \mathrm{s})\left[1+20\left(R_{\odot} / r\right)^{3}\right]^{-1}, n=n_{\odot} U_{0} a_{\odot} /(U a), \mathcal{E}_{\mathrm{W}}=$ $n m_{\mathrm{p}}\left(\delta v_{\odot}\right)^{2}$, and $q_{\perp \mathrm{p}}=q_{\| \mathrm{p}}=0$.

\section{A NUMERICAL EXAMPLE RESEMBLING THE FAST SOLAR WIND}

In this section, we focus on a steady-state solution to Equations (10) through (17) in which the model parameters are set equal to the values listed in Table 1. Our choice for $L_{\perp \odot}$ is motivated by Faraday-rotation measurements along lines of sight passing through the corona and near-Sun solar wind 4 (Hollweg et al. 2010). After choosing the super-radial

\footnotetext{
${ }^{4}$ Hollweg et al. (2010) found that the magnetic fluctuations $\delta B$ in the solarwind model of Cranmer et al. (2007) led to close agreement with the fluctuations in the Faraday rotation of radio transmissions from Helios near superior
}

expansion factors $f_{\max }$ and $R_{1}$, we determine $B_{\odot}$ from Equation (5) and the condition

$$
B(1 \mathrm{AU})=2.83 \mathrm{nT},
$$

which is the mean radial magnetic field strength measured during Ulysses' first polar orbit, scaled to $r=$ $1 \mathrm{AU}$ (McComas et al. 2000). This yields $B_{\odot}=11.8$ Gauss.

TABLE 1

PARAMETERS IN Numerical EXAMPLE

\begin{tabular}{lll}
\hline \hline Quantity & & Value \\
\hline$n_{\odot}$ & $\ldots \ldots \ldots \ldots$ & $10^{8} \mathrm{~cm}^{-3}$ \\
$T_{\odot}$ & $\ldots \ldots \ldots \ldots$ & $7 \times 10^{5} \mathrm{~K}$ \\
$\delta v_{\odot}$ & $\ldots \ldots \ldots \ldots$ & $41.4 \mathrm{~km} / \mathrm{s}$ \\
$f_{\max }$ & $\ldots \ldots \ldots \ldots$. & 9 \\
$R_{1}$ & $\ldots \ldots \ldots \ldots$ & $1.29 R_{\odot}$ \\
$L_{\perp \odot}$ & $\ldots \ldots \ldots \ldots$. & $10^{3} \mathrm{~km}$ \\
$c_{\mathrm{d}}$ & $\ldots \ldots \ldots \ldots$ & 0.75 \\
$c_{2}$ & $\ldots \ldots \ldots \ldots$ & 0.17 \\
$\alpha_{\mathrm{H}}$ & $\ldots \ldots \ldots \ldots$ & 0.75 \\
\hline
\end{tabular}

The resulting numerical solution resembles the fast solar wind in several respects and illustrates the physical processes operating in our model. The density profile is shown in the left panel of Figure 2 Near the Sun, $n$ decreases rapidly with increasing $r$. At larger $r$, as the wind approaches its asymptotic speed, $n$ becomes roughly proportional to $r^{-2}$. The Coulomb collision time scales $v_{\mathrm{e}}^{-1}, v_{\mathrm{pp}, \mathrm{C}}^{-1}$, and $v_{\mathrm{pe}}^{-1}$ are shown in the middle panel of Figure 2. Also plotted are the advection time

conjunction. A key parameter in the model of Cranmer et al. (2007) was $L_{\perp}$, which was set equal to $75[(1470 \text { Gauss }) / B]^{1 / 2}$. For $B=11.8$ Gauss (the value of $B_{\odot}$ in our numerical solutions), this leads to $L_{\perp}=837 \mathrm{~km}$, which we have rounded to $10^{3} \mathrm{~km}$ in choosing the value of $L_{\perp \odot \odot}$. It is possible that $L_{\perp \odot}$ is significantly larger than this value, but in this case $\delta B$ would have to be significantly smaller than in the model of Cranmer et al. (2007) in order to be consistent with the Faraday rotation measurements, preventing AW turbulence from providing the heating needed to power the solar wind. We exclude the possibility that $L_{\perp \odot} \ll 10^{3} \mathrm{~km}$, because then $\delta B$ would have to be much larger than in work of Cranmer et al. (2007) in order to be consistent with the Faraday rotation fluctuations, causing $F_{\mathrm{w}}$ to be much greater than the total energy flux of the solar wind. 
scale $t_{\mathrm{adv}}=r / U$ and the total proton scattering time scale $v_{\mathrm{p}}^{-1}$, which includes the effects of temperature-anisotropy instabilities. Close to the coronal base, the density is sufficiently large that Coulomb collisions play an important role, acting to maintain $T_{\mathrm{e}} \simeq T_{\perp \mathrm{p}} \simeq T_{\| \mathrm{p}}$. Farther from the Sun, however, Coulomb collisions cause only a negligible amount of protonelectron energy exchange and proton temperature isotropization. In the right panel of Figure 2, we plot the ratios of thermal to magnetic pressure, $\beta_{\mathrm{e}}, \beta_{\perp \mathrm{p}}$, and $\beta_{\| \mathrm{p}}$ (defined in the figure caption), which vary from values $\ll 1$ near the Sun to values larger than 1 at $1 \mathrm{AU}$.

The profiles of the wind speed $U$ and Alfvén speed $v_{\mathrm{A}}$ are shown in the left panel of Figure 3 , along with the rms amplitude of the fluctuating AW velocity

$$
\delta v_{\mathrm{rms}}=\sqrt{\frac{\mathcal{E}_{\mathrm{w}}}{\rho}} .
$$

In writing Equation (58), we have ignored the energy associated with AWs propagating towards the Sun in the solar-wind frame, so that $\delta v_{\text {rms }}=z_{\text {rms }}^{+} / 2$. In our numerical solution, the Alfvén critical point occurs at

$$
r_{\mathrm{A}}=11.7 R_{\odot},
$$

$U\left(r_{\mathrm{A}}\right)=598 \mathrm{~km} / \mathrm{s}$, and $U(1 \mathrm{AU})=800 \mathrm{~km} / \mathrm{s}$. The AW fluctuations lead to a turbulent heating rate per unit mass $Q / \rho$ that is shown in the middle panel of Figure 3

The partitioning of the turbulent heating power between $Q_{\mathrm{e}}$, $Q_{\perp \mathrm{p}}$, and $Q_{\| \mathrm{p}}$ is shown in the right panel of Figure 3. At $r<$ $1.35 R_{\odot}$, electrons absorb most of the dissipated AW/KAW energy because $\delta v_{\text {rms }}$ and hence $\varepsilon_{\mathrm{p}}$ are comparatively small (making stochastic heating weak) and because $\beta_{\| \mathrm{p}} \ll 1$ (making proton Landau damping and proton transit-time damping weak). Between $r=1.35 R_{\odot}$ and $r=185 R_{\odot}$, stochastic heating is the most efficient dissipation mechanism in the model, and $Q_{\perp \mathrm{p}}>0.5 Q$. At $r>185 R_{\odot}, Q_{\mathrm{e}}$ is the largest of the three heating rates. The parallel proton heating rate remains $<Q_{\mathrm{e}}$ at large $r$ despite the fact that $\beta_{\| \mathrm{p}} \gtrsim 1$ and $\gamma_{\mathrm{p}}>\gamma_{\mathrm{e}}$. The reason for this is that at large $r$ a significant fraction of the turbulent energy cascades to perpendicular scales $\ll \rho_{\text {p }}$ at which the fluctuations dissipate on the electrons.

In Figure 4 we plot $T_{\mathrm{e}}, T_{\perp \mathrm{p}}$, and $T_{\| \mathrm{p}}$, and in Figure 5 we plot the heating rates that help determine these temperature profiles. Between $R_{\odot}$ and $2 R_{\odot}$, there are ripples in the plots of $Q_{\mathrm{e}}$ and $Q$, which result from local flattenings in the Alfvén speed profile (see Equations (42) and (43)) The ripples in $Q_{\mathrm{e}}$ cause $T_{\mathrm{e}}$ and $q_{\mathrm{e}}$ to vary in such a way that the electron conductive heating rate partially offsets the variations in $Q_{\mathrm{e}}$. This can be seen in left panel of Figure 5, in which the electron conductive heating rate $-\nabla \cdot q_{\mathrm{e}}$ is positive at $r>2.25 R_{\odot}$ but alternates sign at each sharp dip in the plot of $\left|\nabla \cdot q_{\mathrm{e}}\right|$. At $R_{\odot}<r \lesssim 2.5 R_{\odot}$, AW turbulence is the dominant heat source for electrons, as the electron heat flux acts primarily to cool the electrons in this region. The left panel of Figure 5 indicates that a significant fraction of the turbulent heating power deposited into the electrons between $R_{\odot}$ and $2.5 R_{\odot}$ is conducted away to either larger or smaller radii. At $r \gtrsim 3 R_{\odot}, Q_{\mathrm{e}}$

\footnotetext{
${ }^{5}$ The oscillations in $Q$ and $Q_{\mathrm{e}}$ between $r=R_{\odot}$ and $r \simeq 2 R_{\odot}$ result in part from our approximating $z_{\mathrm{rms}}^{-}$and $Q$ in Equations (42) and (43) based on the local value of $\left|\partial v_{\mathrm{A}} / \partial r\right|$. A more realistic treatment would account for the fact that $z^{-}$AWs propagate some distance along the magnetic field before their energy cascades and dissipates, so that the local values of $z_{\text {rms }}^{-}$and $Q$ depend upon the value of $\left|\partial v_{\mathrm{A}} / \partial r\right|$ throughout some range of radii.
}

and $\left|\nabla \cdot \boldsymbol{q}_{\mathrm{e}}\right|$ are of similar magnitude, although $\left|\nabla \cdot \boldsymbol{q}_{\mathrm{e}}\right| / Q_{\mathrm{e}}$ grows to a value $\sim 2$ as $r$ increases to $1.2 \mathrm{AU}$. At $r \geq 100 R_{\odot}$ the electrons approach the state described by Hollweg (1976), in which collisionless heat flux is the only source of electron heating and

$$
T_{\mathrm{e}} \propto n^{2 /\left[3\left(1+\alpha_{\mathrm{H}}\right)\right]} .
$$

However, the $T_{\mathrm{e}}$ profile remains slightly flatter than the scaling in Equation (60) because of turbulent heating.

At $2 R_{\odot} \lesssim r<71 R_{\odot}, T_{\perp \mathrm{p}}$ is determined by a balance between turbulent heating and solar-wind expansion, with the proton heat flux and collisions playing only a minor role, as shown in the middle panels of Figures 2 and 5 The quantity $H_{\perp \mathrm{p}}$ plotted in Figure 5 is the perpendicular heating rate resulting from the proton heat flux,

$$
H_{\perp \mathrm{p}}=-\frac{1}{a^{2}} \frac{\partial}{\partial r}\left(a^{2} q_{\perp \mathrm{p}}\right) .
$$

The $T_{\perp \mathrm{p}}$ profile at $2 R_{\odot} \lesssim r<71 R_{\odot}$ is also affected by the self-limiting nature of stochastic heating. As $T_{\perp \mathrm{p}}$ increases, the fluctuations in the electrostatic potential energy at the proton-gyroradius scale become a smaller fraction of the average perpendicular kinetic energy per proton, $k_{\mathrm{B}} T_{\perp \mathrm{p}}$. As a consequence, these fluctuations have less effect on the proton gyro-motion, and the proton orbits become less stochastic. This leads to a strong reduction in the stochastic heating rate when $T_{\perp \mathrm{p}}$ exceeds a certain threshold that depends upon $\delta v_{\mathrm{p}}$ and $c_{2}$, as described in more detail by Chandran (2010). At $r=71 R_{\odot}$, the plasma encounters the threshold of the oblique firehose instability, causing $v_{p}$ to increase abruptly and leading to sharp cusps in the $T_{\perp \mathrm{p}}$ and $T_{\| \mathrm{p}}$ profiles plotted in Figure 4. At $r>71 R_{\odot}$ the temperature anisotropy ratio $T_{\perp \mathrm{p}} / T_{\| \mathrm{p}}$ evolves approximately along the oblique-firehose instability threshold, as shown in the right panel of Figure 4.

The parallel proton heating rate associated with the proton heat flux,

$$
H_{\| \mathrm{p}}=-\frac{1}{a} \frac{\partial}{\partial r}\left(a q_{\| \mathrm{p}}\right)+\frac{q_{\perp \mathrm{p}}}{a} \frac{\partial a}{\partial r},
$$

is shown in the right panel of Figure 5, along with $Q_{\| \mathrm{p}}$. Although $\left|H_{\| \mathrm{p}}\right|$ is small compared to $Q$, it is larger than $Q_{\| \mathrm{p}}$ at $r \lesssim 70 R_{\odot}$. Despite the fact that $\left|H_{\| \mathrm{p}}\right| \ll Q$, the proton heat flux causes $T_{\| \mathrm{p}}$ to increase with increasing $r$ within this range of radii. This is possible because solar-wind expansion has only a small effect on $T_{\| \mathrm{p}}$ at these radii. As can be seen from Equation (14), solar-wind expansion acts to make $T_{\| \mathrm{p}} \propto n^{2} / B^{2}$ in the absence of competing effects. As the solar wind approaches its asymptotic speed, $n$ becomes approximately proportional to $r^{-2}$. Likewise, when $r$ exceeds a few $R_{\odot}, B \propto r^{-2}$, at least in our model in which solar rotation is neglected (see Section 5.1). When both $n$ and $B$ are $\propto r^{-2}$, (double) adiabatic expansion neither increases nor decreases $T_{\| \mathrm{p}}$.

The electron Coulomb mean free path $\lambda_{\text {mfp }}$ and electron heat fluxes $q_{\mathrm{e}}$ are plotted in the left and middle panels of Figure 6. The electron heat flux transitions from the collisional regime to the collisionless regime in our model at $r=r_{\mathrm{H}}=$ $5 R_{\odot}$, approximately the point at which $\lambda_{\mathrm{mfp}}=r / 2$ as in the collisionless-heat-flux model of Hollweg (1974, 1976). In the collisionless regime, $q_{\mathrm{e}}$ is smaller than the Spitzer-Härm heat flux $q_{\mathrm{e}, \mathrm{S}}$ and somewhat smaller than the free-streaming heat flux

$$
q_{\mathrm{sat}, \mathrm{e}}=1.5 n k_{\mathrm{B}} T_{\mathrm{e}} v_{\mathrm{te}}
$$



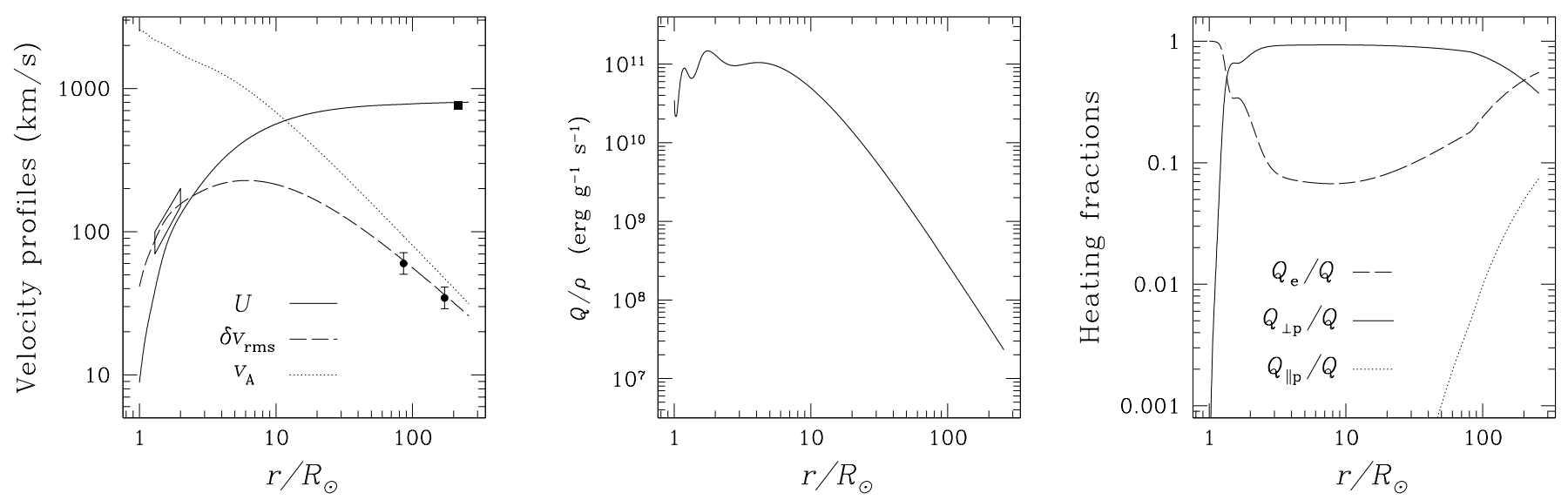

FIG. 3.- Left panel: The open box represents the range of upper limits on $\delta v_{\text {rms }}$ obtained by Esser et al. (1999). The filled circles are Helios measurements of $\delta v_{\text {rms }}$ (Bavassano et al. 2000). The filled square is the median proton flow speed $U=761 \mathrm{~km} / \mathrm{s}$ at heliographic latitudes $>36^{\circ}$ during Ulysses' first polar orbit, scaled to $r=1 \mathrm{AU}$ (McComas et al. 2000). The size of this square represents the range $702 \mathrm{~km} / \mathrm{s}<U<803 \mathrm{~km} / \mathrm{s}$ corresponding to the $5^{\text {th }}$ through $95^{\text {th }}$ percentiles of the measured distribution. Middle panel: Total turbulent heating rate per unit mass. Right panel: The fractions of the turbulent heating power that go to electron heating, perpendicular proton heating, and parallel proton heating.
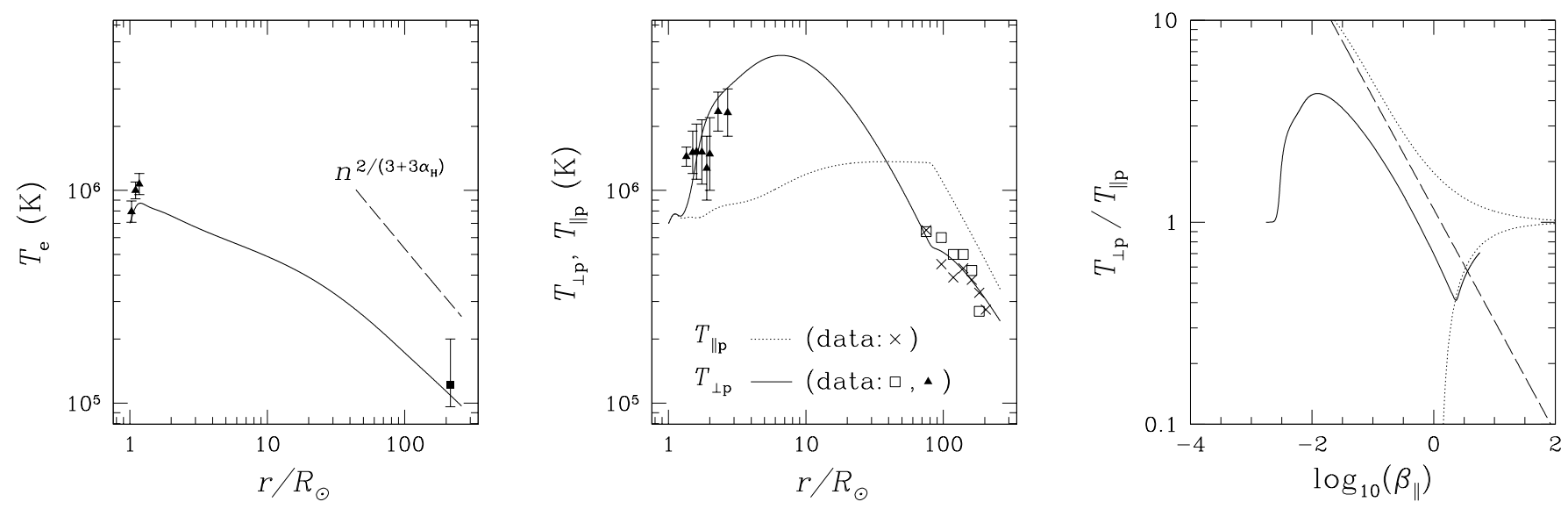

FIG. 4.-Left panel: Triangles are electron temperatures inferred from spectroscopic observations of a polar coronal hole (Landi 2008). The square is the mean electron temperature in ISEE 3 and Ulysses measurements of fast-wind streams with $600 \mathrm{~km} / \mathrm{s}<U<700 \mathrm{~km} / \mathrm{s}$ (Newbury et al. 1998). Middle panel: Triangles are proton kinetic temperatures inferred from UVCS measurements of a polar coronal hole (Esser et al.|1999). Open squares ( $\times$ s) are perpendicular (parallel) proton temperatures measured by Helios in fast solar-wind streams with $700 \mathrm{~km} / \mathrm{s}<U<800 \mathrm{~km} / \mathrm{s}$ (Marsch et al. 1982b). Right panel: The value of $T_{\perp \mathrm{p}} / T_{\| \mathrm{p}}$ in our numerical solution progresses from left to right along the solid-line curve as $r$ increases from $1 R_{\odot}$ to $258 R_{\odot}$. The dotted lines give the instability thresholds for the mirror instability (upper curve) and oblique firehose instability (lower curve) from Hellinger et al. (2006), at which the maximum instability growth rates equal $10^{-3} \Omega_{\mathrm{p}}$, where $\Omega_{\mathrm{p}}$ is the proton cyclotron frequency. The long-dashed line is the fit $T_{\perp \mathrm{p}} / T_{\| \mathrm{p}}=1.16 \beta_{\| \mathrm{p}}^{-0.553}$ obtained by Marsch et al. (2004) to Helios data of high-speed wind streams between $r=0.29 \mathrm{AU}$ and $r=0.98 \mathrm{AU}$.

but comparable to Helios measurements of the electron heat flux in the fast solar wind (Marsch \& Richter 1984).

The proton heat fluxes are plotted in the right panel of Figure 6 As this figure shows, $q_{\perp \mathrm{p}}$ and $q_{\| \mathrm{p}}$ are significantly smaller than the free-streaming heat flux

$$
q_{\mathrm{sat}, \mathrm{p}}=1.5 n k_{\mathrm{B}} T_{\mathrm{p}} v_{\mathrm{tp}},
$$

where $v_{\mathrm{tp}}=\sqrt{k_{\mathrm{B}} T_{\mathrm{p}} / m_{\mathrm{p}}}$. This can be understood on a qualitative level from the following argument. If a proton temperature gradient were set up in a collisionless plasma with no background flow and no initial heat flux, then the proton heat flux would grow in time, approaching a level comparable to the free-streaming value after a time $\sim t_{\text {cross }}=l_{\mathrm{T}} / v_{\mathrm{tp}}$, where $l_{\mathrm{T}}=T_{\mathrm{p}} /\left|\nabla T_{\mathrm{p}}\right|$. In our model, $l_{\mathrm{T}} \sim r$, and this "crossing time scale" is a factor of $\sim M$ larger than the expansion time scale of the solar wind, $t_{\mathrm{adv}}=r / U$, where $M=U / v_{\mathrm{tp}}$ is the Mach number. In our numerical solution, $M$ equals 3.56 at $r=10 R_{\odot}$ and grows monotonically with increasing $r$ to a value of 15.1 at $r=215 R_{\odot}$. Thus, throughout most of our solution, $t_{\mathrm{adv}}=r / U \ll t_{\text {cross }}$. As a result, the protons in our model do not have time to set up a heat flux comparable to the free-streaming heat flux within the time it takes for the plasma to double its distance from the Sun, which reduces $q_{\perp \mathrm{p}}$ and $q_{\| \mathrm{p}}$ relative to their values in a stationary plasma with comparable density and temperature profiles.

As discussed in Section 2 the total energy flowing through the flux tube in our model per unit time, $a F_{\text {tot }}$, is independent of $r$ in steady state. (In our numerical solution, the ratio of the maximum to minimum values of $a F_{\text {tot }}$ is 1.003.) This makes it straightforward to identify the principal source of energy in our model and to understand how energy is converted from one form to another as plasma flows away from the Sun. The total energy flux defined in Equation (27) is the sum of the bulk-flow kinetic energy flux

$$
F_{\mathrm{U}}=\frac{1}{2} \rho U^{3}
$$



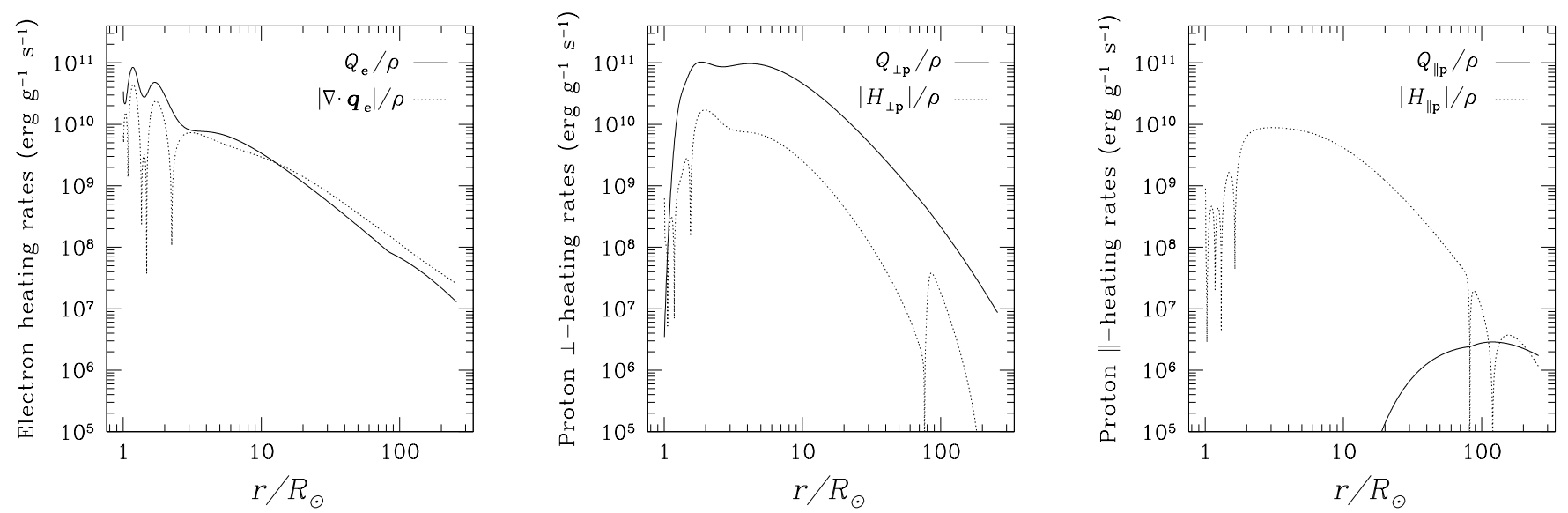

FIG. 5.- Heating rates associated with the heat fluxes and the dissipation of AW/KAW turbulence.
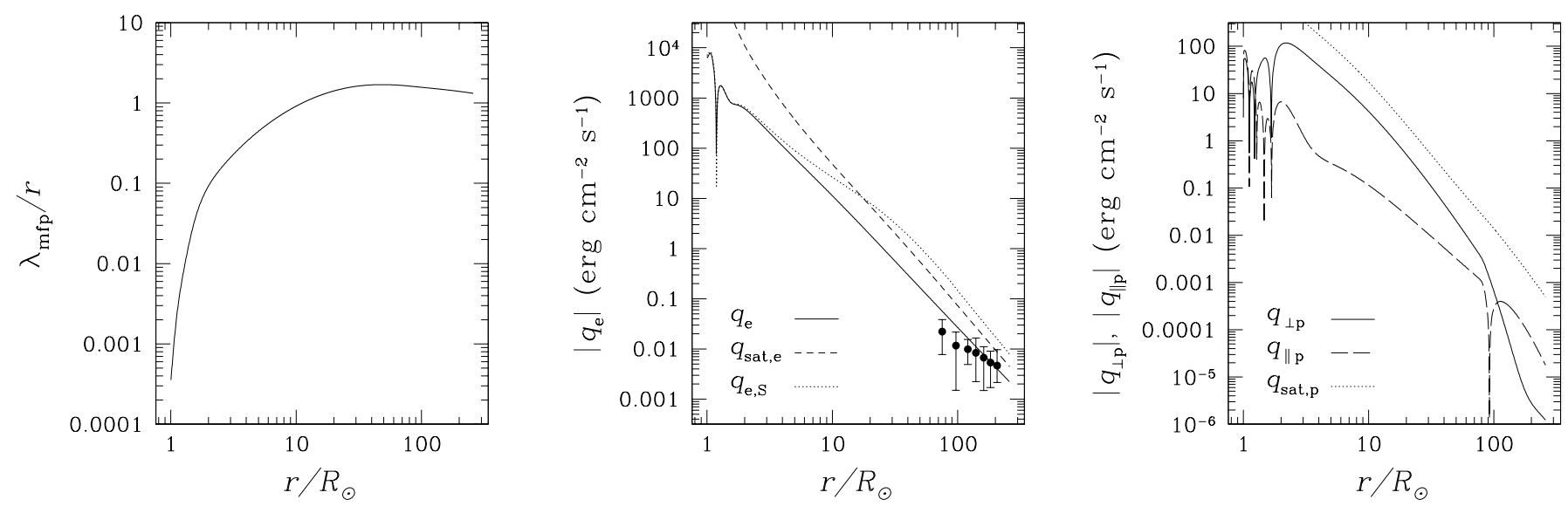

FIG. 6. - Left panel: $\lambda_{\mathrm{mfp}}$ is the electron Coulomb mean free path. Middle panel: The electron heat flux $q_{\mathrm{e}}$, free-streaming heat flux $q_{\text {sat.e }}$, and Spitzer-Härm heat flux $q_{\mathrm{e}, \mathrm{S}}$. The circles are Helios measurements of the electron heat flux in high-speed wind with $U>600 \mathrm{~km} / \mathrm{s}$ (Marsch \& Richter 1984). Right panel: The proton heat fluxes $q_{\perp \mathrm{p}}$ and $q_{\| \mathrm{p}}$ and free-streaming heat flux $q_{\mathrm{sat}, \mathrm{p}}$.

the gravitational potential energy flux

$$
F_{\mathrm{g}}=-\frac{U G M_{\odot} \rho}{r}
$$

the enthalpy flux

$$
F_{\mathrm{e}}=U n k_{\mathrm{B}}\left(\frac{5 T_{\mathrm{e}}}{2}+T_{\perp \mathrm{p}}+\frac{3 T_{\| \mathrm{p}}}{2}\right),
$$

the total heat flux

$$
q_{\mathrm{tot}}=q_{\mathrm{e}}+q_{\perp \mathrm{p}}+q_{\| \mathrm{p}}
$$

and the AW enthalpy flux

$$
F_{\mathrm{w}}=\left(\frac{3 U}{2}+v_{\mathrm{A}}\right) \mathcal{E}_{\mathrm{w}} .
$$

We plot these fluxes in Figure 7 normalized to the total energy flux $F_{\text {tot. }}$. As this figure shows, the wind in this solution is driven fundamentally by the AW enthalpy flux. As the plasma flows away from the Sun, part of the AW enthalpy flux is converted into gravitational potential energy flux as the flow lifts material out of the Sun's gravitational potential well. Most of the remaining AW enthalpy flux is gradually converted into bulk-flow kinetic energy flux, which dominates the total energy flux at $r=1 \mathrm{AU}$.

\section{DISCUSSION}

In this section, we discuss our results and present a second steady-state solution that incorporates pitch-angle scattering by the cyclotron instability.

\subsection{Solar Rotation}

If solar rotation were taken into account, $\boldsymbol{B}$ would follow the Parker spiral, and at large distances from the axis of rotation $B$ would become approximately azimuthal rather than radial, with $B \propto r^{-1}$ instead of $B \propto r^{-2}$. Assuming $n \propto r^{-2}$, double adiabatic expansion in this azimuthal-field regime would lead to the scalings $T_{\perp \mathrm{p}} \propto r^{-1}, T_{\| \mathrm{p}} \propto r^{-2}$, and $T_{\perp \mathrm{p}} / T_{\| \mathrm{p}} \propto r$. In contrast, in the radial magnetic field of our model, double adiabatic expansion leads to $T_{\perp \mathrm{p}} \propto r^{-2}, T_{\| \mathrm{p}} \propto r^{0}$, and $T_{\perp \mathrm{p}} / T_{\| \mathrm{p}} \propto r^{-2}$ assuming $n \propto r^{-2}$. The inclusion of rotation would thus increase the temperature anisotropy ratio $T_{\perp \mathrm{p}} / T_{\| \mathrm{p}}$ at large $r$. In the solar wind, the transition between radial and azimuthal magnetic field occurs gradually throughout a 


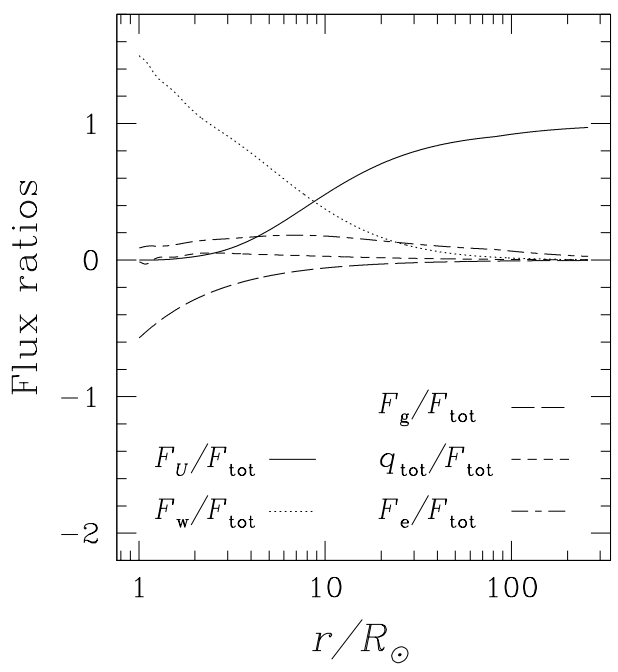

FIG. 7.- The fractions of the total energy flux $F_{\text {tot }}$ that come from the bulk-flow kinetic energy flux $F_{\mathrm{U}}$, the $\mathrm{AW}$ enthalpy flux $F_{\mathrm{w}}$, the gravitational potential energy flux $F_{\mathrm{g}}$, the total heat flux $q_{\mathrm{tot}}$, and the enthalpy flux $F_{\mathrm{e}}$.

range of radii centered at $r_{\perp} \sim U / \Omega$, where $\Omega$ is the angular frequency of the Sun's rotation, and $r_{\perp}$ is distance from the Sun's spin axis. For fast wind with $U=800 \mathrm{~km} / \mathrm{s}$, and for $\Omega=2.64 \times 10^{-6} \mathrm{~s}^{-1}$ (the value of $\Omega$ at a solar latitude of $45^{\circ}$ (Snodgrass \& Ulrich 1990) $), U / \Omega=1.4 \mathrm{AU}$.

In addition to modifying the temperature anisotropy ratio at large $r$, the inclusion of rotation would increase the total magnetic field strength at large $r$, thereby reducing $\beta_{\| \mathrm{p}}$. For example, during the first polar orbit of Ulysses, the ratio of the mean total field strength (scaled to $r=1 \mathrm{AU}$ ) to the mean radial field strength (scaled to $r=1 \mathrm{AU}$ ) was $\simeq 1.7$ (McComas et al. 2000). At fixed $n$ and $T_{\| \mathrm{p}}$, increasing $B$ by a factor of $\simeq 1$.7 reduces $\beta_{\| \mathrm{p}}$ by a factor of $\simeq 3$. Our overestimate of $\beta_{\| \mathrm{p}}$ at $r \sim 1$ AU causes us to overestimate $Q_{\| \mathrm{p}}$ and also pushes the thresholds of the firehose and mirror instabilities towards smaller temperature anisotropies.

\subsection{The Need for Parallel Proton Cooling}

In the numerical solution presented in Section $4, T_{\| \mathrm{p}}>T_{\perp \mathrm{p}}$ at $r>35 R_{\odot}$. In contrast, in Helios measurements of fastwind streams with $U>700 \mathrm{~km} / \mathrm{s}, T_{\perp \mathrm{p}}$ typically exceeds $T_{\| \mathrm{p}}$ between $r=60 R_{\odot}$ and $r=130 R_{\odot}$ (Marsch et al. 1982b). Part of this discrepancy may be due to our neglect of solar rotation (Section 5.1) or one or more perpendicular proton heating mechanisms (Section 5.6). This discrepancy may also arise, at least in part, from our neglect of kinetic mechanisms that act to reduce $T_{\| \mathrm{p}}$. For example, part of the parallel proton thermal energy in the fast solar wind is in the form of a proton beam (Marsch et al. 1982b, 2004). When the relative speed of the beam component with respect to the core of the proton distribution exceeds $\sim v_{\mathrm{A}}$, the proton beam excites plasma instabilities that slow the beam down (Daughton \& Gary 1998). Since $v_{A}$ decreases with increasing $r$, these instabilities lead to the steady deceleration of the proton beam component, which reduces the parallel proton thermal energy (Hellinger \& Trávníček 2011). The possible need for parallel proton cooling in the solar wind was previously suggested by Hu et al. (1997).

\subsection{Uncertainties in the Total Turbulent Heating Rate}

Two of the assumptions in our estimate of $Q$ become increasingly inaccurate as $r$ increases. First, we have assumed that $z^{-} \ll z^{+}$, i.e., that most of the AWs propagate away from the Sun in the solar-wind frame. While $z_{\text {rms }}^{+}$is likely $\gg z_{\mathrm{rms}}^{-}$in coronal holes (Cranmer \& van Ballegooijen 2005; Verdini \& Velli 2007; Cranmer 2010), Helios measurements in the fast solar wind show that $z_{\mathrm{rms}}^{+} / z_{\mathrm{rms}}^{-}$decreases from $\simeq 4$ to $\simeq 2$ as $r$ increases from $80 R_{\odot}$ to 1 AU (Bavassano et al. 2000). Second, the model of Chandran \& Hollweg (2009) that we employ to estimate $z_{\text {rms }}^{-}$assumes that the $z^{-}$energy cascade time at the outer scale, $t_{\text {casc }}^{-} \simeq L_{\perp} / z_{\text {rms }}^{+}$, is much shorter than the linear wave period $P$. For the numerical solution presented in Section $4, t_{\text {casc }}^{-}$grows steadily as $r$ increases, reaching a value $\simeq 3 \times 10^{3} \mathrm{~s}$ at $r=100 R_{\odot}$ and a value $\simeq 10^{4} \mathrm{~s}$ at $r=200 R_{\odot}$. Thus, for $P \sim 1$ hour, the assumption that $t_{\text {casc }}^{-} \ll P$ breaks down at large $r$. We also note that velocity shear may be an important additional source of AW turbulence in the solar wind, one that is not included in our model (Roberts et al. 1987). As a source of both $z^{+}$and $z^{-}$ fluctuations, AW excitation by velocity shear acts to decrease the ratio $z_{\mathrm{rms}}^{+} / z_{\mathrm{rms}}^{-}$in the solar wind (Roberts et al. 1992).

\subsection{Uncertainties in the Division of the Turbulent Heating Power Between $Q_{\mathrm{e}}, Q_{\perp \mathrm{p}}$, and $Q_{\| \mathrm{p}}$.}

Our prescription for partitioning the turbulent heating power between protons and electrons, and between parallel and perpendicular proton heating, depends strongly upon two of the assumptions we have made in modeling AW turbulence in the solar wind: the scaling of $\delta v_{\mathrm{p}}$ in Equation (48) and the "critical balance" condition in Equation (46). Equation (48) corresponds to an assumption that the inertialrange velocity power spectrum is $\propto k_{\perp}^{-3 / 2}$, which is consistent with spacecraft measurements at $r \stackrel{\perp}{=} 1 \mathrm{AU}$ (Podesta et al. 2007; Podesta \& Bhattachariee 2010; Chen et al. 2011) and direct numerical simulations of AW turbulence in which the fluctuating magnetic field is $\lesssim 0.2$ times the background magnetic field (Maron \& Goldreich 2001; Müller \& Grappin 2005; Mason et al. 2006; Perez \& Boldyrev 2008), including simulations that account for cross helicity, in which $z_{\mathrm{rms}}^{+}>z_{\mathrm{rms}}^{-}$(Perez \& Boldyrev 2009). The power spectrum of the magnetic field is typically steeper $\left(\sim k^{-5 / 3}\right)$ than the velocity power spectrum in spacecraft measurements (Matthaeus \& Goldstein 1982; Goldstein et al. 1995; Bruno \& Carbone 2005; Podesta et al. 2007; Chen et al. 2011) and direct numerical simulations (Müller \& Grappin 2005; Boldyrev et al.2011). The stochastic heating rate in our model, however, depends upon the velocity power spectrum, not the magnetic power spectrum, because the velocity spectrum is a measure of the electric-field fluctuations, which control the stochastic heating rate when $\beta_{\| \mathrm{p}} \lesssim 1$ (Chandran et al. 2010). On the other hand, AW turbulence near the Sun is strongly affected by non-WKB wave reflection and may differ from AW turbulence at $r \sim 1$ AU and from the homogeneous turbulence in the numerical simulations mentioned above (Verdini et al. 2009). If the velocity spectrum is steeper (flatter) than we have assumed, then the amplitude of the velocity fluctuations at $k_{\perp} \rho_{\mathrm{p}} \sim 1$ is smaller (larger) than in Equation (48), and stochastic heating is weaker (stronger) than in our model.

The critical-balance condition that we have adopted in Equation (46) is the same as the condition in Boldyrev's 
(2006) theory of scale-dependent dynamic alignment and is consistent with Perez \& Boldyrev's (2009) extension of this theory to the cross-helical case, which holds that the cascade times of $z^{+}$and $z^{-}$fluctuations are the same even when $z_{\mathrm{rms}}^{+} \neq z_{\mathrm{rms}}^{-}$. Equation (46) is also consistent with the work of Podesta \& Bhattacharjee (2010) if the ratio $p / q$ in their analysis is set equal to unity. However, Equation (46) is not consistent with three competing (and mutually inconsistent) models of AW turbulence with cross helicity (Lithwick et al. 2007; Beresnyak \& Lazarian 2008; Chandran 2008a). Moreover, none of the six studies just mentioned accounted for the non-WKB reflection of AWs, which could affect wavenumber anisotropy in the solar wind. The range of parallel wavenumbers that are present at $k_{\perp} \rho_{\mathrm{p}}=1$ in AW turbulence in the solar wind is thus uncertain. If the typical values of $\left|k_{\|}\right|$are larger than we have assumed, then linear wave damping is stronger than we have assumed, implying that $Q_{\perp \mathrm{p}} / Q$ is smaller than in our model. In addition, near $r=1 \mathrm{AU}$, increasing the linear damping rates would increase $Q_{\| \mathrm{p}} / Q_{\mathrm{e}}$, because less power would cascade to scales $\ll \rho_{\mathrm{p}}$, and because protons absorb most of the power that is dissipated at $k_{\perp} \rho_{\mathrm{p}} \sim 1$ when $\beta_{\mathrm{p}}>1$.

Other sources of uncertainty in our partitioning of the turbulent heating power include the stochastic-heating constant $c_{2}$ in Equation (50), which is not known for the case of protons interacting with strong AW/KAW turbulence. If we have underestimated $c_{2}$, then we have overestimated the efficiency of stochastic heating, causing our model to overestimate $T_{\perp \mathrm{p}}$. In addition, the use of linear Vlasov theory to estimate damping rates may be inaccurate when applied to largeamplitude AW/KAW turbulence in the solar wind (Borovsky \& Gary 2011; but see also Lehe et al. 2009).

\subsection{The Coronal Electron Temperature}

Electron temperatures in the low corona inferred from line ratios are found to be in the range $\sim 8 \times 10^{5}-$ $10^{6} \mathrm{~K}$ (Habbal et al. 1993; Doschek et al. 2001; Wilhelm 2006; Landi 2008), similar to the electron temperature in our model. On the other hand, Ulysses measurements of ion charge states in the fast solar wind emanating from the south polar coronal hole suggest that $T_{\mathrm{e}}$ reaches a maximum of $\simeq 1.5 \times 10^{6} \mathrm{~K}$ at $r \sim 1.3-1.5 R_{\odot}$ ( Ko et al. 1997). The numerical solution we have presented in Section 4 does not reach such high electron temperatures, which may indicate that we have under-estimated the electron heating rate. On the other hand, the electron temperatures inferred from ion charge-state ratios at $1 \mathrm{AU}$ may be inflated to some degree by the presence of superthermal electrons in the corona, which could significantly enhance the rates of ionization into highenergy charge states (Owocki \& Scudder 1983; Burgi 1987; Ko et al. 1996), potentially making the $T_{\mathrm{e}}$ profile in the left panel of Figure 4 consistent with the ion-charge-state measurements.

\subsection{Other Possible Heating Mechanisms}

Although low-frequency AW turbulence is the only nonconductive heating mechanism in our model, other mechanisms may be important in the solar wind. For example, compressive waves are believed to play an important role in chromospheric heating, and may deposit a significant amount of energy in the low corona as well (Cranmer et al. 2007; Verdini et al. 2010). Type-II spicules may also be an important source of heating in the low corona (De Pontieu et al. 2011). Farther from the Sun, the solar wind may be heated by high-frequency waves that are generated by either a turbulent cascade (Leamon et al. 1998; Hollweg \& Isenberg 2002; Hamilton et al. 2008; Isenberg \& Vasquez 2011) or by instabilities driven by the differential flow between the core of the proton velocity distribution and either alpha particles or proton beams (Gary et al. 2000; Hellinger \& Trávníček 2011).

\subsection{Cyclotron Instability versus Mirror Instability}

Hellinger et al. (2006) showed that near $r=1 A U$, the proton temperature anisotropy ratio $T_{\perp \mathrm{p}} / T_{\| \mathrm{p}}$ is limited from above by the threshold of the mirror instability. However, using Helios measurements, Bourouaine et al. (2010) found that $T_{\perp \mathrm{p}} / T_{\| \mathrm{p}}$ appears to be limited from above by the cyclotron instability in fast solar wind streams between $0.3 \mathrm{AU}$ and 0.4 AU. To investigate how this latter possibility would affect our numerical solutions, we have repeated the numerical calculation presented in Section 4 with the parameter values listed in Table 1 using a model for enhanced proton pitchangle scattering based on the cyclotron instability instead of the mirror instability. That is, we have set $R_{\mathrm{m}} \rightarrow R_{\mathrm{c}}$ in Equation (38), with (Hellinger et al. 2006)

$$
R_{\mathrm{c}}=1+0.43\left(\beta_{\| \mathrm{p}}+0.0004\right)^{-0.42} .
$$

The density and temperature profiles in this second steadystate solution are shown in Figure 8 . The density profile is very similar to the profile in the left panel of Figure 2. The profiles of $T_{\mathrm{e}}$ and $T_{\perp \mathrm{p}}$ are very similar to the profiles shown in the left and middle panels of Figure 4 The most notable difference between the temperature profiles in the two numerical solutions is in $T_{\| \mathrm{p}}$, which is larger at $4 R_{\odot} \lesssim r \lesssim 10 R_{\odot}$ when the cyclotron instability threshold is used. As can be seen in the right panel of Figure 8 , when $T_{\perp \mathrm{p}} / T_{\| \mathrm{p}}$ is limited from above by the anisotropy ratio in Equation (70), the plasma encounters and then evolves approximately along the cyclotroninstability threshold at small $r$ and small $\beta_{\| \mathrm{p}}$. At larger $r$ and larger $\beta_{\| \mathrm{p}}$, the plasma evolves approximately along the threshold of the oblique firehose instability, as in the numerical solution presented in Section 4

\subsection{Sharp Boundaries in Parameter Space}

We have found that there are regions in parameter space in which tiny changes in certain parameters lead to large changes in the final steady-state solutions. This phenomenon may be related to the abrupt transition from fast wind to slow wind in theoretical models in which the magnetic geometry varies with heliographic latitude (Cranmer 2005; Cranmer et al. 2007).

\subsection{Comparison to Previous Studies}

A number of authors have developed solar-wind models incorporating temperature anisotropy (Leer \& Axford 1972; Whang 1972; Demars \& Schunk 1991; Hu et al.|1997; Olsen \& Leer 1999; Endeve \& Leer 2001; Lie-Svendsen et al. 2001; Janse et al. 2006), and some of these models also included energy and/or momentum deposition by AWs (Hu et al. 1997; Olsen \& Leer 1999; Lie-Svendsen et al. 2001). Our work, however, has several features that are not present in these previous studies. First, we evaluate the total turbulent heating rate $Q$ using an analytical theory of lowfrequency $\mathrm{AW}$ turbulence driven by non-WKB wave reflection (Dmitruk et al. 2002; Chandran \& Hollweg 2009). Second, we divide the total turbulent heating rate $Q$ into three 

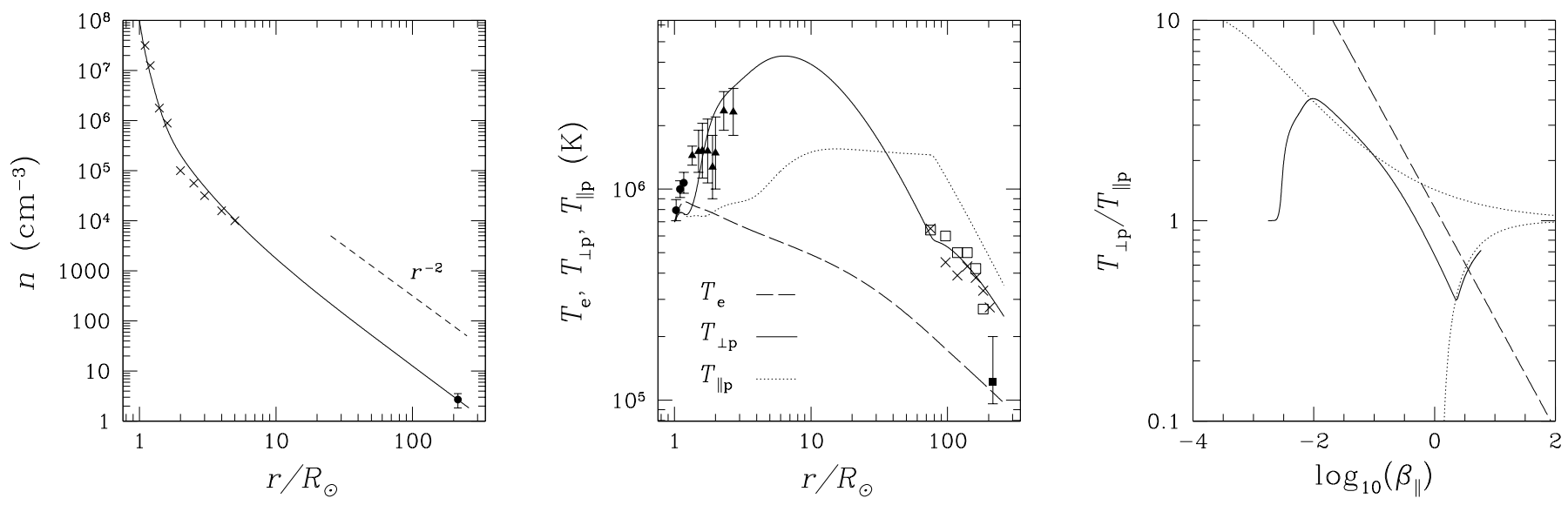

FIG. 8. - Steady-state numerical solution when $T_{\perp \mathrm{p}} / T_{\| \mathrm{p}}$ is limited from above by the threshold of the cyclotron instability (Equation 70 ) rather than the mirror instability. The parameter values for this solution are given in Table 1 Left panel: Same as left panel of Figure 2 Middle panel: Symbols have same meaning as in the middle panel of Figure 4 In addition, the circles are electron temperatures inferred from spectroscopic observations of a polar coronal hole (Landi 2008), and the filled square is the mean electron temperature in ISEE 3 and Ulysses measurements of fast-wind streams with $600 \mathrm{~km} / \mathrm{s}<U<700 \mathrm{~km} / \mathrm{s}$ (Newbury et al. 1998). Right panel: Same as right panel of Figure 4 except that the upper dotted-line curve is the instability threshold for the cyclotron instability in Equation (70).

parts $\left(Q_{\mathrm{e}}, Q_{\perp \mathrm{p}}\right.$ and $\left.Q_{\| \mathrm{p}}\right)$ using an analytical model of the collisionless dissipation of low-frequency AW turbulence. Third, we do not include any heating other than that which is provided by the proton and electron heat fluxes and lowfrequency AW turbulence. Fourth, we account for the mirror (or cyclotron) and oblique firehose instabilities by enhancing the proton pitch-angle scattering rate when the proton temperature anisotropy exceeds the threshold of either instability.

Two other groups of authors have developed solar-wind models based on energy and momentum deposition by lowfrequency AW turbulence driven by non-WKB wave reflection (Cranmer et al. 2007; Verdini et al. 2010). These authors allowed for a broad spectrum of AW frequencies at the coronal base and accounted for the fact that higher-frequency waves undergo less reflection. In contrast, our estimates for $z_{\mathrm{rms}}^{-}$and $Q$ in Equations (42) and (43) were obtained in the low-frequency limit (Chandran \& Hollweg 2009). On the other hand, these models treated the solar wind as a single fluid, without distinguishing between proton and electron temperatures. Also, neither model incorporated temperature anisotropy.

\section{CONCLUSION}

We have developed a two-fluid model of the solar wind that accounts for proton temperature anisotropy, pitch-angle scattering from mirror (or cyclotron) and firehose instabilities, and (kinetic) Alfvén Wave (AW/KAW) turbulence. We neglect rotation and consider solar wind flowing along a narrow magnetic flux tube centered on a radial magnetic field line. The turbulent heating in our model is partitioned between electrons and protons, and between perpendicular and parallel proton heating, in accord with recent results on stochastic ion heating (Chandran et al. 2010) and linear damping rates calculated from the full hot-plasma dispersion relation. The electron heat flux in our model transitions from the Spitzer-Härm value in the collisional region near the Sun to the Hollweg (1976) value in the nearly collisionless conditions at larger $r$. To evaluate the proton heat flux in the presence of temperature anisotropy in both collisional and collisionless conditions, we use a fourth-velocity-moment fluid closure based on the guiding-center Vlasov equation (Kulsrud 1983; Snyder et al. 1997). Our model conserves energy, and in steady state the total energy flowing through the cross section of the flux tube becomes independent of $r$. No energy is added to the solution through ad hoc heating terms.

In Section 4 we present a steady-state numerical solution to our model equations for $R_{\odot}<r<1.2 \mathrm{AU}$, which we analyze in detail in order to gain insight into the different physical processes operating within the model. As shown in Figure 7, it is the AW enthalpy flux $F_{\mathrm{w}}$ that drives the solar wind in this solution. As plasma flows away from the Sun, the AW enthalpy flux is gradually converted into gravitational potential energy flux and bulk-flow kinetic energy flux. By the time the flow reaches $1 \mathrm{AU}$, the total energy flux is dominated by the bulk-flow kinetic energy. As shown in Figure 5] electrons are heated primarily by the dissipation of AW/KAW turbulence at $r \leq 2.5 R_{\odot}$. At $r \gtrsim 3 R_{\odot}$, AW turbulence and conduction provide comparable amounts of electron heating, to within a factor of $\sim 2$. Between $1.35 R_{\odot}$ and $185 R_{\odot}$, AW/KAW turbulence dissipates primarily via stochastic proton heating, leading to substantial perpendicular proton heating, as shown in the right panel of Figure 3. Parallel proton heating via Landau and transit-time damping accounts for $<10 \%$ of the total turbulent heating at all radii in this solution, and $Q_{\| \mathrm{p}}<0.01 Q$ at $r<100 R_{\odot}$. The proton heat fluxes $q_{\perp \mathrm{p}}$ and $q_{\| \mathrm{p}}$ cause $T_{\| \mathrm{p}}$ to increase gradually as $r$ increases from $2 R_{\odot}$ to $\sim 20 R_{\odot}$, despite the fact that solar-wind expansion reduces $q_{\perp \mathrm{p}}$ and $q_{\| \mathrm{p}}$ relative to their free-streaming values. At $r=71 R_{\odot}$, the plasma encounters the threshold of the oblique firehose instability, causing the proton pitch-angle scattering rate to increase. At $r>71 R_{\odot}$, the proton temperature anisotropy ratio evolves approximately along the firehose-instability threshold as $\beta_{\| \mathrm{p}}$ increases.

This numerical solution is broadly consistent with a number of observations, as illustrated in Figures 2, 3, 4, and 6, supporting the idea that AW turbulence may be one of the primary mechanisms responsible for heating coronal holes and accelerating the solar wind (Parker 1965; Coleman 1968). Perhaps the most notable achievement of our model is that it comes close to explaining observations of perpendicular proton tem- 
peratures, even though the heating in the model is provided by low-frequency AW turbulence rather than resonant cyclotron interactions. The main uncertainties in our results are associated with the stochastic heating rate in strong AW/KAW turbulence, the wavenumber anisotropy $\left(k_{\|} / k_{\perp}\right)$ in reflectiondriven AW/KAW turbulence, the total turbulent heating rate at large $r$, and the effects of solar rotation on the temperature anisotropy ratio $T_{\perp \mathrm{p}} / T_{\| \mathrm{p}}$.

One of our principal objectives in this work has been to to connect theoretical studies of microphysical processes with observations of macrophysical quantities in the solar wind. By comparing our model to observations, we have been able to obtain a new test of the viability of AW turbulence as a mechanism for heating the solar wind and coronal holes. At this point, the results of this test are encouraging, but not fully conclusive, because of the uncertainties described above. However, as our understanding of kinetic plasma physics and turbulence in the solar wind progresses, it will be possible to use models such as the one we have developed to obtain increasingly rigorous tests of competing theories and, ultimately, to gain greater insight into coronal heating and the origin of the solar wind.

We thank Steve Cranmer, Joe Hollweg, Greg Howes, Phil Isenberg, Yuan-Kuen Ko, Jean Perez, and Jason Tenbarge for helpful discussions. This work was supported in part by grant NNX11AJ37G from NASA's Heliophysics Theory Program, NSF grant AGS-0851005, NSF/DOE grant AGS-1003451, DOE grant DE-FG02-07-ER46372, NSF/DOE grant PHY0812811, and NSF grant ATM-0752503.

\section{APPENDIX}

\section{FOURTH-MOMENT FLUID CLOSURE OF THE GUIDING-CENTER VLASOV EQUATION}

The derivation of the equations in our model begins with Kulsrud's formulation of collisionless MHD for a proton-electron plasma (Kulsrud 1983). Kulsrud's approach was to expand all quantities in the Vlasov and Maxwell equations in powers of $1 / e$, where $e$ is the proton charge, and to consider the limit $e \rightarrow \infty$. This limit corresponds to the case in which the Debye length $\lambda_{\mathrm{D}}$ and proton gyroradius $\rho_{\mathrm{p}}$ are much smaller than the length scales over which the macroscopic quantities vary appreciably. The fundamental variables in Kulsrud's theory are the mass density $\rho$, the fluid velocity $\boldsymbol{U}$ (which is the same for electrons and protons to lowest order in $1 / e$ ), the magnetic field $\boldsymbol{B}$, the proton and electron distribution functions $f_{\mathrm{p}}$ and $f_{\mathrm{e}}$, and the parallel component of the electric field $\boldsymbol{E}$, given by $E_{\|}=\hat{\boldsymbol{b}} \cdot \boldsymbol{E}$, where $\hat{\boldsymbol{b}}=\boldsymbol{B} / \boldsymbol{B}$. To lowest order in $1 / e$, these variables satisfy the equations

$$
\begin{gathered}
\frac{\partial \rho}{\partial t}+\nabla \cdot(\rho \boldsymbol{U})=0 \\
\rho\left(\frac{\partial \boldsymbol{U}}{\partial t}+\boldsymbol{U} \cdot \boldsymbol{\nabla} \boldsymbol{U}\right)=\frac{(\boldsymbol{\nabla} \times \boldsymbol{B}) \times \boldsymbol{B}}{4 \pi}-\nabla \cdot \boldsymbol{P} \\
\frac{\partial \boldsymbol{B}}{\partial t}=\nabla \times(\boldsymbol{U} \times \boldsymbol{B}) \\
\boldsymbol{P}=\sum_{s} p_{\perp s}(\boldsymbol{I}-\hat{\boldsymbol{b}} \hat{\boldsymbol{b}})+\sum_{s} p_{\| s} \hat{\boldsymbol{b}} \hat{\boldsymbol{b}} \\
p_{\perp s}=\frac{m_{s}}{2} \int f_{s}\left|\boldsymbol{v}-v_{\|} \hat{\boldsymbol{b}}-\boldsymbol{v}_{E}\right|^{2} d^{3} v \\
p_{\| s}=m_{s} \int f_{s}\left(v_{\|}-U_{\|}\right)^{2} d^{3} v \\
\sum_{s} e_{s} n_{s}=0 \\
n_{s}=\int f_{s} d^{3} v
\end{gathered}
$$

and (see Snyder et al. 1997)

$$
\frac{\partial}{\partial t}\left(f_{\mathrm{s}} B\right)+\nabla \cdot\left[f_{\mathrm{s}} B\left(v_{\|} \hat{\boldsymbol{b}}+\boldsymbol{v}_{E}\right)\right]+\frac{\partial}{\partial v_{\|}}\left[f_{\mathrm{s}} B\left(-\hat{\boldsymbol{b}} \cdot \frac{D \boldsymbol{v}_{E}}{D t}-\mu \hat{\boldsymbol{b}} \cdot \nabla B+\frac{e_{s} E_{\|}}{m_{s}}\right)\right]=0
$$

where $s$ is a subscript indicating particle species (p for proton and e for electron), $f_{S}$ is the distribution function of particle species $s$, $m_{s}$ and $e_{s}$ are the mass and charge of species $s, \boldsymbol{v}$ is particle velocity, $v_{\|}=\hat{\boldsymbol{b}} \cdot \boldsymbol{v}, \boldsymbol{v}_{E}=c(\boldsymbol{E} \times \boldsymbol{B}) / B^{2}, \boldsymbol{\mu}=\left|\boldsymbol{v}-v_{\|} \hat{\boldsymbol{b}}-\boldsymbol{v}_{E}\right|^{2} / 2 B$, $U_{\|}=\boldsymbol{U} \cdot \hat{\boldsymbol{b}}$, and $D / D t=\partial / \partial t+\left(v_{\|} \hat{\boldsymbol{b}}+\boldsymbol{v}_{E}\right) \cdot \nabla$. In Equation A9], $f_{s}$ is regarded as a function of position $\boldsymbol{x}$, time $t$, magnetic moment $\mu$, and parallel velocity $v_{\|}$. Rather than retain the subscripts on the number densities, we define

$$
n=n_{\mathrm{p}}
$$

which is also equal to $n_{\mathrm{e}}$ because of Equation (A7). We have neglected the electron contribution to the mass density, setting $\rho=n m_{\mathrm{p}}$. The parallel and perpendicular temperatures are related to the parallel and perpendicular pressures defined above in the usual way: $p_{\perp \mathrm{s}}=n k_{\mathrm{B}} T_{\perp \mathrm{s}}$ and $p_{\| \mathrm{s}}=n k_{\mathrm{B}} T_{\| \mathrm{s}}$. 
Snyder et al. (1997) extended Kulsrud's collisionless MHD to account for collisions by adding a BGK collision operator (Gross \& Krook 1956) to the right-hand side of Equation (A9). For the case we consider here, in which the electrons and ions have the same average velocity $U$ and number density $n$, this collision operator takes the form

$$
C\left(f_{s}\right)=\sum_{k} v_{s k}\left(F_{M s}-f_{s}\right)
$$

where

$$
F_{M s}=n\left(\frac{m_{s}}{2 \pi k_{\mathrm{B}} T_{\mathrm{s}}}\right)^{3 / 2} \exp \left[-\frac{m_{\mathrm{s}}\left(v_{\|}-U_{\|}\right)^{2}}{2 k_{\mathrm{B}} T_{s}}-\frac{m_{s} \mu B}{k_{\mathrm{B}} T_{s}}\right]
$$

is a shifted Maxwellian with temperature

$$
T_{s}=\frac{2 T_{\perp s}+T_{\| s}}{3}
$$

and $v_{s k}$ is the collision frequency for momentum exchange between species $s$ and species $k$. (Here, we neglect energy exchange between protons and electrons, but we include it in section 2]) Snyder et al. (1997) then obtained a hierarchy of fluid equations by multiplying Equation (A9) by various powers of $v_{\|}$and $\mu$ and then integrating over $v_{\|}$and $\mu$. For the protons, the equations for $p_{\perp \mathrm{p}}$ and $p_{\| \mathrm{p}}$ can be written (Snyder et al. 1997; Sharma et al. 2006)

$$
\rho B \frac{d}{d t}\left(\frac{p_{\perp \mathrm{p}}}{\rho B}\right)=-\nabla \cdot\left(q_{\perp \mathrm{p}} \hat{\boldsymbol{b}}\right)-q_{\perp \mathrm{p}} \nabla \cdot \hat{\boldsymbol{b}}+\frac{v_{\mathrm{p}}}{3}\left(p_{\| \mathrm{p}}-p_{\perp \mathrm{p}}\right)
$$

and

$$
\frac{\rho^{3}}{2 B^{2}} \frac{d}{d t}\left(\frac{B^{2} p_{\| \mathrm{p}}}{\rho^{3}}\right)=-\nabla \cdot\left(q_{\| \mathrm{p}} \hat{\boldsymbol{b}}\right)+q_{\perp \mathrm{p}} \nabla \cdot \hat{\boldsymbol{b}}+\frac{v_{\mathrm{p}}}{3}\left(p_{\perp \mathrm{p}}-p_{\| \mathrm{p}}\right),
$$

where

$$
\begin{gathered}
q_{\perp \mathrm{p}}=m_{\mathrm{p}} \int f_{\mathrm{p}} \mu B\left(v_{\|}-U_{\|}\right) d^{3} v, \\
q_{\| \mathrm{p}}=\frac{m_{\mathrm{p}}}{2} \int f_{\mathrm{p}}\left(v_{\|}-U_{\|}\right)^{3} d^{3} v,
\end{gathered}
$$

$m_{\mathrm{p}}$ is the proton mass, and

$$
v_{\mathrm{p}}=v_{\mathrm{pp}}+\mathrm{v}_{\mathrm{pe}}
$$

In Section 2 we neglect the $v_{\text {pe }}$ term in Equation (A18) because it is smaller than the proton-proton Coulomb collision frequency. Our $q_{\| \mathrm{p}}$ is by definition a factor of 2 smaller than Snyder et al.'s (1997). In this appendix, the Lagrangian time derivative is given by

$$
\frac{d}{d t}=\frac{\partial}{\partial t}+\boldsymbol{U} \cdot \nabla
$$

which generalizes Equation (9) to allow for arbitrary flow velocities. The equations for $q_{\perp \mathrm{p}}$ and $q_{\| \mathrm{p}}$ are

$$
\rho^{2} \frac{d}{d t}\left(\frac{q_{\perp \mathrm{p}}}{\rho^{2}}\right)+v_{\mathrm{p}} q_{\perp \mathrm{p}}=-\nabla \cdot\left(r_{\| \perp} \hat{\boldsymbol{b}}\right)+\frac{p_{\perp \mathrm{p}}}{\rho} \hat{\boldsymbol{b}} \cdot \nabla p_{\| \mathrm{p}}+\left[\frac{p_{\perp \mathrm{p}}\left(p_{\| \mathrm{p}}-p_{\perp \mathrm{p}}\right)}{\rho}+r_{\perp \perp}-r_{\| \perp}\right] \nabla \cdot \hat{\boldsymbol{b}}
$$

and

$$
\frac{\rho^{4}}{B^{3}} \frac{d}{d t}\left(\frac{B^{3} q_{\| \mathrm{p}}}{\rho^{4}}\right)+v_{\mathrm{p}} q_{\| \mathrm{p}}=-\frac{1}{2} \nabla \cdot\left(r_{\|\| \|} \hat{\boldsymbol{b}}\right)+\frac{3 p_{\| \mathrm{p}}}{2 \rho} \hat{\boldsymbol{b}} \cdot \nabla p_{\| \mathrm{p}}+\frac{3}{2}\left[\frac{p_{\| \mathrm{p}}\left(p_{\| \mathrm{p}}-p_{\perp \mathrm{p}}\right)}{\rho}+r_{\| \perp}\right] \nabla \cdot \hat{\boldsymbol{b}}
$$

where

$$
\begin{gathered}
r_{\perp \perp}=m_{\mathrm{p}} \int f_{\mathrm{p}} \mu^{2} B^{2} d^{3} v \\
r_{\| \perp}=m_{\mathrm{p}} \int f_{\mathrm{p}} \mu B\left(v_{\|}-U_{\|}\right)^{2} d^{3} v
\end{gathered}
$$

and

$$
r_{\|\| \|}=m_{\mathrm{p}} \int f_{\mathrm{p}}\left(v_{\|}-U_{\|}\right)^{4} d^{3} v
$$

In Equation (A20), we have corrected a minor error in Equation (19) of Snyder et al. (1997): the fourth term on the left-hand side of their Equation (19) should be $q_{\perp s} \boldsymbol{\nabla} \cdot \boldsymbol{U}$ instead of $q_{\perp \mathrm{s}} \boldsymbol{\nabla} \cdot\left(U_{\|} \hat{\boldsymbol{b}}\right)$. 
To close these fluid equations, we set $f_{\mathrm{p}}=F_{B M}$ in Equations (A22) through (A24), where

$$
F_{B M}=\frac{n m_{\mathrm{p}}^{3 / 2}}{\left(2 \pi k_{\mathrm{B}}\right)^{3 / 2} T_{\perp \mathrm{p}} T_{\| \mathrm{p}}^{1 / 2}} \exp \left[-\frac{m_{\mathrm{p}} \mu B}{k_{\mathrm{B}} T_{\perp p}}-\frac{m_{\mathrm{p}}\left(v_{\|}-U_{\|}\right)^{2}}{2 k_{\mathrm{B}} T_{\| \mathrm{p}}}\right]
$$

is a bi-Maxwellian distribution with the same values of $n, U_{\|}, T_{\perp \mathrm{p}}$ and $T_{\| \mathrm{p}}$ as the protons. This enables us to rewrite Equations (A20) and (A21) as

$$
\rho^{2} \frac{d}{d t}\left(\frac{q_{\perp \mathrm{p}}}{\rho^{2}}\right)+v_{\mathrm{p}} q_{\perp \mathrm{p}}=-\frac{n k_{\mathrm{B}}^{2} T_{\| \mathrm{p}}}{m_{\mathrm{p}}} \hat{\boldsymbol{b}} \cdot \nabla T_{\perp \mathrm{p}}+\frac{n k_{\mathrm{B}}^{2} T_{\perp \mathrm{p}}\left(T_{\perp \mathrm{p}}-T_{\| \mathrm{p}}\right)}{m_{\mathrm{p}}} \nabla \cdot \hat{\boldsymbol{b}}
$$

and

$$
\frac{\rho^{4}}{B^{3}} \frac{d}{d t}\left(\frac{B^{3} q_{\| \mathrm{p}}}{\rho^{4}}\right)+v_{\mathrm{p}} q_{\| \mathrm{p}}=-\frac{3 n k_{\mathrm{B}}^{2} T_{\| \mathrm{p}}}{2 m_{\mathrm{p}}} \hat{\boldsymbol{b}} \cdot \nabla T_{\| \mathrm{p}} .
$$

Equations (A26) and (A27) were derived in different ways and with differing treatments of collisions by Endeve \& Leer (2001), Lie-Svendsen et al. (2001), and Ramos (2003).

For the electrons, we set $T_{\perp \mathrm{e}}=T_{\| \mathrm{e}}=T_{\mathrm{e}}$ as described in section 2 and close the electron fluid equations by specifying the electron heat flux $\boldsymbol{q}_{\mathrm{e}}$ in terms of lower moments of the electron distribution (Section 2.1). The electrons then satisfy a standard energy equation,

$$
\frac{3}{2} n^{5 / 3} k_{\mathrm{B}} \frac{d}{d t}\left(\frac{T_{\mathrm{e}}}{n^{2 / 3}}\right)=-\nabla \cdot \boldsymbol{q}_{\mathrm{e}}
$$

In Section 2, we adapt the general equations given in this appendix to our 1D solar-wind model and add extra terms to incorporate the effects of AW turbulence, collisional energy exchange between protons and electrons, and temperature isotropization by firehose and mirror instabilities.

\section{REFERENCES}

Alazraki, G., \& Couturier, P. 1971, A\&A, 13, 380

Allen, C. W. 1973, Astrophysical Quantities, 3rd ed., ed. Allen, C. W. (London: University of London, Athlone Press)

Antonucci, E., Dodero, M. A., \& Giordano, S. 2000, Sol. Phys., 197, 115

Bale, S. D., Kasper, J. C., Howes, G. G., Quataert, E., Salem, C., \& Sundkvist, D. 2009, Physical Review Letters, 103, 211101

Bale, S. D., Kellogg, P. J., Mozer, F. S., Horbury, T. S., \& Reme, H. 2005, Physical Review Letters, 94, 215002

Barnes, A. 1966, Physics of Fluids, 9, 1483

Bavassano, B., Pietropaolo, E., \& Bruno, R. 2000, J. Geophys. Res., 105, 15959

Belcher, J. W., \& Davis, Jr., L. 1971, J. Geophys. Res., 76, 3534

Beresnyak, A., \& Lazarian, A. 2008, ApJ, 682, 1070

Boldyrev, S. 2006, Physical Review Letters, 96, 115002

Boldyrev, S., Perez, J. C., Borovsky, J. E., \& Podesta, J. J. 2011, ArXiv e-prints

Book, D. L. 1983, NRL (Naval Research Laboratory) plasma formulary, revised, Tech. rep.

Borovsky, J. E., \& Gary, S. P. 2011, Journal of Geophysical Research (Space Physics), 116, A07101

Bourouaine, S., Marsch, E., \& Neubauer, F. M. 2010, Geophys. Res. Lett., 37, L14104

Bourouaine, S., Marsch, E., \& Vocks, C. 2008, ApJ, 684, L119

Braginskii, S. I. 1965, in Reviews of Plasma Physics, ed. M. A. Leontovich, Vol. 1 (New York: Consultants Bureau), 205

Bruno, R., \& Carbone, V. 2005, Living Reviews in Solar Physics, 2, 4

Burgi, A. 1987, J. Geophys. Res., 92, 1057

Chandran, B. D. G. 2005, Physical Review Letters, 95, 265004

-. 2008a, ApJ, 685, 646

—. 2008b, Physical Review Letters, 101, 235004

-. 2010, ApJ, 720, 548

Chandran, B. D. G., \& Hollweg, J. V. 2009, ApJ, 707, 1659

Chandran, B. D. G., Li, B., Rogers, B. N., Quataert, E., \& Germaschewski, K. 2010, ApJ, 720, 503

Chandran, B. D. G., Quataert, E., Howes, G. G., Xia, Q., \&

Pongkitiwanichakul, P. 2009, ApJ, 707, 1668

Chen, C. H. K., Bale, S. D., Salem, C., \& Mozer, F. S. 2011, ArXiv e-prints

Chen, L., Lin, Z., \& White, R. 2001, Physics of Plasmas, 8, 4713

Chew, G. F., Goldberger, M. L., \& Low, F. E. 1956, Proceedings of the Royal Society of London A, 236, 112

Cho, J., \& Lazarian, A. 2004, ApJ, 615, L41

Coleman, P. J. 1968, ApJ, 153, 371
Cranmer, S. R. 2005, in ESA Special Publication, Vol. 592, Solar Wind 11/SOHO 16, Connecting Sun and Heliosphere, ed. B. Fleck,

T. H. Zurbuchen, \& H. Lacoste, 159-+

Cranmer, S. R. 2010, ApJ, 710, 676

Cranmer, S. R., Matthaeus, W. H., Breech, B. A., \& Kasper, J. C. 2009, ApJ, 702,1604

Cranmer, S. R., \& van Ballegooijen, A. A. 2003, ApJ, 594, 573

-. 2005, 156, 265

Cranmer, S. R., van Ballegooijen, A. A., \& Edgar, R. J. 2007, ApJS, 171, 520

Daughton, W., \& Gary, S. P. 1998, J. Geophys. Res., 103, 20613

De Pontieu, B., et al. 2007, Science, 318, 1574

- 2011, Science, 331, 55

Demars, H. G., \& Schunk, R. W. 1991, Planet. Space Sci., 39, 435

Dewar, R. L. 1970, Physics of Fluids, 13, 2710

Dmitruk, P., Matthaeus, W. H., Milano, L. J., Oughton, S., Zank, G. P., \& Mullan, D. J. 2002, ApJ, 575, 571

Dmitruk, P., Matthaeus, W. H., \& Seenu, N. 2004, ApJ, 617, 667

Doschek, G. A., Feldman, U., Laming, J. M., Schühle, U., \& Wilhelm, K. 2001, ApJ, 546, 559

Endeve, E., \& Leer, E. 2001, Sol. Phys., 200, 235

Esser, R., Fineschi, S., Dobrzycka, D., Habbal, S. R., Edgar, R. J., Raymond, J. C., Kohl, J. L., \& Guhathakurta, M. 1999, ApJ, 510, L63

Forman, M. A., Wicks, R. T., \& Horbury, T. S. 2011, ApJ, 733, 76

Galtier, S., Nazarenko, S. V., Newell, A. C., \& Pouquet, A. 2000, Journal of Plasma Physics, 63, 447

Gary, S. P., Yin, L., Winske, D., \& Reisenfeld, D. B. 2000,

Geophys. Res. Lett., 27, 1355

Goldreich, P., \& Sridhar, S. 1995, ApJ, 438, 763

Goldstein, M. L., Roberts, D. A., \& Matthaeus, W. H. 1995, ARA\&A, 33, 283

Gomberoff, L., Gnavi, G., \& Gratton, F. T. 1996, J. Geophys. Res., 101, 13517

Gross, E. P., \& Krook, M. 1956, Physical Review, 102, 593

Gruzinov, A. V. 1998, ApJ, 501, 787

Habbal, S. R., Esser, R., \& Arndt, M. B. 1993, ApJ, 413, 435

Hamilton, K., Smith, C. W., Vasquez, B. J., \& Leamon, R. J. 2008, Journal of Geophysical Research (Space Physics), 113, 1106

Harmon, J. K., \& Coles, W. A. 2005, Journal of Geophysical Research (Space Physics), 110, 3101

Hartle, R. E., \& Sturrock, P. A. 1968, ApJ, 151, 1155

Heinemann, M., \& Olbert, S. 1980, J. Geophys. Res., 85, 1311 
Hellinger, P., \& Trávníček. 2011, submitted to J. Geophys. Res.

Hellinger, P., Trávníček, P., Kasper, J. C., \& Lazarus, A. J. 2006, Geophys. Res. Lett., 33, 9101

Higdon, J. C. 1984, ApJ, 285, 109

Hollweg, J. V. 1973a, ApJ, 181, 547

-. 1973b, J. Geophys. Res., 78, 3643

—. 1974, J. Geophys. Res., 79, 3845

-. 1976, J. Geophys. Res., 81, 1649

Hollweg, J. V., Bird, M. K., Volland, H., Edenhofer, P., Stelzried, C. T., \& Seidel, B. L. 1982, J. Geophys. Res., 87, 1

Hollweg, J. V., Cranmer, S. R., \& Chandran, B. D. G. 2010, ApJ, 722, 1495

Hollweg, J. V., \& Isenberg, P. A. 2002, Journal of Geophysical Research (Space Physics), 107, 1147

Holzer, T. E., \& Leer, E. 1980, J. Geophys. Res., 85, 4665

Horbury, T. S., Forman, M., \& Oughton, S. 2008, Physical Review Letters, 101,175005

Hossain, M., Gray, P. C., Pontius, D. H., Matthaeus, W. H., \& Oughton, S. 1995, Physics of Fluids, 7, 2886

Howes, G. G., Cowley, S. C., Dorland, W., Hammett, G. W., Quataert, E., \& Schekochihin, A. A. 2008a, Journal of Geophysical Research (Space Physics), 113, 5103

Howes, G. G., Dorland, W., Cowley, S. C., Hammett, G. W., Quataert, E., Schekochihin, A. A., \& Tatsuno, T. 2008b, Physical Review Letters, 100, 065004

Hu, Y. Q., Esser, R., \& Habbal, S. R. 1997, J. Geophys. Res., 102, 14661

Iroshnikov, P. S. 1963, AZh, 40, 742

Isenberg, P. A., \& Vasquez, B. J. 2011, ApJ, 731, 88

Janse, A. M., Lie-Svendsen, Ø., \& Leer, E. 2006, Physica Scripta Volume T, 122,66

Johnson, J. R., \& Cheng, C. Z. 2001, Geophys. Res. Lett., 28, 4421

Kadomtsev, B. B., \& Pogutse, O. P. 1974, Soviet Journal of Experimental and Theoretical Physics, 38, 283

Kasper, J. C., Lazarus, A. J., \& Gary, S. P. 2002, Geophys. Res. Lett., 29, 170000

Ko, Y., Fisk, L. A., Geiss, J., Gloeckler, G., \& Guhathakurta, M. 1997, Sol. Phys., 171, 345

Ko, Y.-K., Fisk, L. A., Gloeckler, G., \& Geiss, J. 1996, Geophys. Res. Lett., 23, 2785

Kohl, J. L., et al. 1998, ApJ, 501, L127

Kopp, R. A., \& Holzer, T. E. 1976, Sol. Phys., 49, 43

Kraichnan, R. H. 1965, Physics of Fluids, 8, 1385

Kulsrud, R. 1983, in Handbook of Plasma Physics, ed. M. N. Rosenbluth \& R. Z. Sagdeev (New York: North Holland), 115

Landi, E. 2008, ApJ, 685, 1270

Leamon, R. J., Matthaeus, W. H., Smith, C. W., \& Wong, H. K. 1998, ApJ, 507, L181

Leamon, R. J., Smith, C. W., Ness, N. F., \& Wong, H. K. 1999

J. Geophys. Res., 104, 22331

Leer, E., \& Axford, W. I. 1972, Sol. Phys., 23, 238

Lehe, R., Parrish, I. J., \& Quataert, E. 2009, ApJ, 707, 404

Li, X., Habbal, S. R., Kohl, J., \& Noci, G. 1998, ApJ, 501, L133+

Lie-Svendsen, Ø., Leer, E., \& Hansteen, V. H. 2001, J. Geophys. Res., 106, 8217

Lithwick, Y., Goldreich, P., \& Sridhar, S. 2007, ApJ, 655, 269

MacBride, B. T., Smith, C. W., \& Forman, M. A. 2008, ApJ, 679, 1644

Maron, J., \& Goldreich, P. 2001, ApJ, 554, 1175

Marsch, E., Ao, X.-Z., \& Tu, C.-Y. 2004, Journal of Geophysical Research (Space Physics), 109, 4102

Marsch, E., \& Richter, A. K. 1984, J. Geophys. Res., 89, 6599

Marsch, E., Schwenn, R., Rosenbauer, H., Muehlhaeuser, K., Pilipp, W., \& Neubauer, F. M. 1982b, J. Geophys. Res., 87, 52

Mason, J., Cattaneo, F., \& Boldyrev, S. 2006, Physical Review Letters, 97, 255002

Matthaeus, W. H., \& Goldstein, M. L. 1982, J. Geophys. Res., 87, 6011

Matthaeus, W. H., Zank, G. P., Oughton, S., Mullan, D. J., \& Dmitruk, P. 1999, ApJ, 523, L93

McChesney, J. M., Stern, R. A., \& Bellan, P. M. 1987, Physical Review Letters, 59, 1436
McComas, D. J., et al. 2000, J. Geophys. Res., 105, 10419

Müller, W., \& Grappin, R. 2005, Physical Review Letters, 95, 114502

Newbury, J. A., Russell, C. T., Phillips, J. L., \& Gary, S. P. 1998,

J. Geophys. Res., 103, 9553

Ng, C. S., \& Bhattacharjee, A. 1996, ApJ, 465, 845

Olsen, E. L., \& Leer, E. 1999, J. Geophys. Res., 104, 9963

Owocki, S. P., \& Scudder, J. D. 1983, ApJ, 270, 758

Parashar, T. N., Shay, M. A., Cassak, P. A., \& Matthaeus, W. H. 2009,

Physics of Plasmas, 16, 032310

Parker, E. N. 1958, ApJ, 128, 664

-. 1965, Space Science Reviews, 4, 666

Perez, J. C., \& Boldyrev, S. 2008, ApJ, 672, L61

—. 2009, Physical Review Letters, 102, 025003

Podesta, J. J. 2009, ApJ, 698, 986

Podesta, J. J., \& Bhattacharjee, A. 2010, ApJ, 718, 1151

Podesta, J. J., Roberts, D. A., \& Goldstein, M. L. 2007, ApJ, 664, 543

Quataert, E. 1998, ApJ, 500, 978

Ramos, J. J. 2003, Physics of Plasmas, 10, 3601

Roberts, D. A., Goldstein, M. L., Klein, L. W., \& Matthaeus, W. H. 1987, J. Geophys. Res., 92, 12023

Roberts, D. A., Goldstein, M. L., Matthaeus, W. H., \& Ghosh, S. 1992

J. Geophys. Res., 971, 17115

Sahraoui, F., Goldstein, M. L., Robert, P., \& Khotyaintsev, Y. V. 2009, Physical Review Letters, 102, 231102

Schekochihin, A. A., Cowley, S. C., Dorland, W., Hammett, G. W., Howes

G. G., Quataert, E., \& Tatsuno, T. 2009, ApJS, 182, 310

Schunk, R. W. 1975, Planet. Space Sci., 23, 437

Sharma, P., Hammett, G. W., Quataert, E., \& Stone, J. M. 2006, ApJ, 637, 952

Shebalin, J. V., Matthaeus, W., \& Montgomery, D. 1983, Journal of Plasma Physics, 29, 525

Smith, C. W., Matthaeus, W. H., Zank, G. P., Ness, N. F., Oughton, S., \&

Richardson, J. D. 2001, J. Geophys. Res., 106, 8253

Snodgrass, H. B., \& Ulrich, R. K. 1990, ApJ, 351, 309

Snyder, P. B., Hammett, G. W., \& Dorland, W. 1997, Physics of Plasmas, 4, 3974

Spangler, S. R. 2002, ApJ, 576, 997

Spitzer, L., \& Härm, R. 1953, Physical Review, 89, 977

Stawarz, J. E., Smith, C. W., Vasquez, B. J., Forman, M. A., \& MacBride,

B. T. 2009, ApJ, 697, 1119

Stix, T. 1992, Waves in Plasmas (New York: Springer-Verlag)

Strauss, H. R. 1976, Physics of Fluids, 19, 134

Suzuki, T. K., \& Inutsuka, S.-i. 2005, ApJ, 632, L49

Tomczyk, S., McIntosh, S. W., Keil, S. L., Judge, P. G., Schad, T., Seeley,

D. H., \& Edmondson, J. 2007, Science, 317, 1192

Tu, C., \& Marsch, E. 1995, Space Science Reviews, 73, 1

Tu, C.-Y. 1987, Sol. Phys., 109, 149

-. 1988, J. Geophys. Res., 93, 7

van Ballegooijen, A. A., Asgari-Targhi, M., Cranmer, S. R., \& DeLuca,

E. E. 2011, ApJ, 736, 3

Velli, M. 1993, A\&A, 270, 304

Velli, M., Grappin, R., \& Mangeney, A. 1989, Physical Review Letters, 63, 1807

Verdini, A., \& Velli, M. 2007, ApJ, 662, 669

Verdini, A., Velli, M., \& Buchlin, E. 2009, ApJ, 700, L39

Verdini, A., Velli, M., Matthaeus, W. H., Oughton, S., \& Dmitruk, P. 2010, ApJ, 708, L116

Voitenko, Y., \& Goossens, M. 2004, ApJ, 605, L149

Whang, Y. C. 1972, ApJ, 178, 221

Wilhelm, K. 2006, A\&A, 455, 697

Yoon, P. H., \& Fang, T.-M. 2008, Plasma Physics and Controlled Fusion, 50, 085007

Zank, G. P., \& Matthaeus, W. H. 1992, Journal of Plasma Physics, 48, 85 